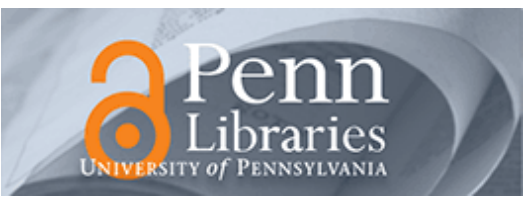

University of Pennsylvania

ScholarlyCommons

$6-2012$

\title{
Colloquium: Disclination loops, point defects, and all that in nematic liquid crystals
}

\author{
Gareth P. Alexander \\ University of Pennsylvania, garetha@sas.upenn.edu \\ Bryan Gin-ge Chen \\ University of Pennsylvania \\ Elisabetta A. Matsumoto \\ University of Pennsylvania, Princeton University \\ Randall Kamien \\ University of Pennsylvania, kamien@physics.upenn.edu
}

Follow this and additional works at: https://repository.upenn.edu/physics_papers

Part of the Physics Commons

\section{Recommended Citation}

Alexander, G. P., Chen, B. G., Matsumoto, E. A., \& Kamien, R. (2012). Colloquium: Disclination loops, point defects, and all that in nematic liquid crystals. Retrieved from https://repository.upenn.edu/

physics_papers/250

Alexander, G. P., Chen, B. G., Matsumoto, E. A., \& Kamien, R. D. (2012). Colloquium: Disclination loops, point defects, and all that in nematic liquid crystals. Reviews of Modern Physics, 84(2), 497-514. doi: 10.1103/

RevModPhys.84.497

(c) 2012 American Physical Society

This paper is posted at ScholarlyCommons. https://repository.upenn.edu/physics_papers/250

For more information, please contact repository@pobox.upenn.edu. 


\title{
Colloquium: Disclination loops, point defects, and all that in nematic liquid crystals
}

\author{
Abstract \\ The homotopy theory of topological defects is a powerful tool for organizing and unifying many ideas \\ across a broad range of physical systems. Recently, experimental progress was made in controlling and \\ measuring colloidal inclusions in liquid crystalline phases. The topological structure of these systems is \\ quite rich but, at the same time, subtle. Motivated by experiment and the power of topological reasoning, \\ the classification of defects in uniaxial nematic liquid crystals was reviewed and expounded upon. \\ Particular attention was paid to the ambiguities that arise in these systems, which have no counterpart in \\ the much-storied XY model or the Heisenberg ferromagnet.

\section{Disciplines} \\ Physical Sciences and Mathematics | Physics

\section{Comments} \\ Alexander, G. P., Chen, B. G., Matsumoto, E. A., \& Kamien, R. D. (2012). Colloquium: Disclination loops, \\ point defects, and all that in nematic liquid crystals. Reviews of Modern Physics, 84(2), 497-514. doi: \\ 10.1103/RevModPhys.84.497 \\ (c) 2012 American Physical Society
}




\title{
Colloquium: Disclination loops, point defects, and all that in nematic liquid crystals
}

\author{
Gareth P. Alexander \\ Department of Physics and Astronomy, University of Pennsylvania, \\ 209 South 33rd Street, Philadelphia, Pennsylvania 19104, USA, and \\ Centre for Complexity Science and Department of Physics, University of Warwick, \\ Coventry, CV4 7AL, United Kingdom \\ Bryan Gin-ge Chen \\ Department of Physics and Astronomy, University of Pennsylvania, \\ 209 South 33rd Street, Philadelphia, Pennsylvania 19104, USA \\ Elisabetta A. Matsumoto \\ Department of Physics and Astronomy, University of Pennsylvania, \\ 209 South 33rd Street, Philadelphia, Pennsylvania 19104, USA \\ Princeton Center for Theoretical Science, Princeton University, \\ Princeton, New Jersey 08544, USA \\ Randall D. Kamien* \\ Department of Physics and Astronomy, University of Pennsylvania, \\ 209 South 33rd Street, Philadelphia, Pennsylvania 19104, USA
}

(published 9 April 2012)

\begin{abstract}
The homotopy theory of topological defects is a powerful tool for organizing and unifying many ideas across a broad range of physical systems. Recently, experimental progress was made in controlling and measuring colloidal inclusions in liquid crystalline phases. The topological structure of these systems is quite rich but, at the same time, subtle. Motivated by experiment and the power of topological reasoning, the classification of defects in uniaxial nematic liquid crystals was reviewed and expounded upon. Particular attention was paid to the ambiguities that arise in these systems, which have no counterpart in the much-storied $X Y$ model or the Heisenberg ferromagnet.

DOI: 10.1103/RevModPhys.84.497

PACS numbers: 61.30. $-\mathrm{v}, 61.72 .-\mathrm{y}, 02.40 . P c$
\end{abstract}

\section{CONTENTS}

I. Introduction

II. Circular Loops Around Defects: The Fundamental Group

A. Two-dimensional director: $\mathbb{R P}^{1}$

B. Making precise measurements

C. Three-dimensional director: $\mathbb{R P}^{2}$

III. Hedgehogs

IV. Disclination Loops

A. Measuring with spheres

B. Measuring with tori

V. Biaxial Nematics and the Odd Hedgehog

VI. Conclusion
497

498

499

500

501

504

507

507

507

510

512

\section{INTRODUCTION}

First identified through the beautiful textures of their defects, liquid crystalline materials may very well be the ideal proving grounds for exploring notions of broken symmetries, associated Goldstone modes, phenomena akin

\footnotetext{
*kamien@physics.upenn.edu
}

to the Higgs mechanism, and, of course, topological defects. Indeed, the subjects of topological defects and liquid crystals are so intertwined that it is difficult to see how a thorough understanding of one could be garnered without knowledge of the other. Moreover, it has proven fruitful to treat the defects as fundamental excitations; solitons in the sine-Gordon model can be treated as interacting fermions (Coleman, 1975), vortices in the $X Y$ model drive the Kosterlitz-Thouless phase transition (Kosterlitz and Thouless, 1973), controlling flux lines is essential for maintaining superconductivity (Nelson, 1988), and the theory of magnetic monopoles shows that they can be viewed as point particles (Coleman, 1988). In all of these cases, the fluctuations and configurations of the smooth background field around the defects can be replaced with an effective interaction between the defects. Thus the elasticity and statistical mechanics of these systems can be recast in terms of a discrete set of topological charges. Because of these successes and physicist's deeply ingrained love for Gauss's law and the connection between flux and charge, we naturally attempt to interpret more complex systems in the same terms. Unfortunately, these cases are exceptions; in this Colloquium we emphasize this important point and attempt 
to clarify if, when, and how it is appropriate to view defects as point charges.

Nematics are a prototypical liquid crystalline material, although a sample will flow like a liquid, owing to the lack of positional order, the rod-shaped, typically organic or aromatic ring molecules are locally aligned, leading to orientational order, with widely applied optical consequences. Defects in a nematic liquid crystal, as in any ordered medium, are places where the nematic order vanishes and the molecular direction is thus ill defined. They occur in the form of isolated points and lines, either system spanning with end points on the boundaries of the sample or closed up into loops. We use as our example the three-dimensional uniaxial nematic, a system that turns out to not be as simple as it might seem. Not only is it, in some sense, the simplest counterexample to the "defects as charges" approach, but it is also now extremely relevant from the experimental point of view as it has been demonstrated that defects can be manipulated in nematic cells with great precision and variety allowing complex links and knots to be tied (Kamien, 2011; Tkalec et al., 2011). Although the history or path dependence of the defect motion at once spoils the program of reducing the configuration space to discrete data, it simultaneously introduces a new set of topological degrees of freedom in the threedimensional nematic. The path dependence imposes a nonAbelian structure to the classification of states. In other liquid crystalline systems, line defects in the biaxial nematic are anticipated to entangle topologically (Poénaru and Toulouse, 1977) and the interaction between disclinations and dislocations in smectics suffers similar path dependence (Poénaru and Toulouse, 1977; Chen, Alexander, and Kamien, 2009). Certain non-Abelian quantum systems have been proposed as a route to topological codes and computation (Kitaev, 2003; Nayak et al., 2008).

Although they arise naturally during phase transitions, defects can also be induced deliberately by local excitation with a laser (Smalyukh et al., 2010) or through boundary conditions as with colloidal inclusions, where the anchoring of molecules at the colloid surface induces the presence of defects in the surrounding liquid crystal. This is an especially rich technique allowing for the generation of a wide range of defects and for studying the interactions between them. For instance, the defect accompanying a colloid may take the form of an isolated point (Poulin et al., 1997) or of a disclination loop encircling the particle in a "Saturn ring" configuration (Terentjev, 1995), while two or more colloids in close proximity can form a variety of "entangled" structures where a single disclination loop wraps around both of them (Guzmán et al., 2003; Ravnik et al., 2007; Ravnik and Žumer, 2009). These last situations realize the interaction between line and point defects that is a central aspect of this Colloquium.

In Sec. II we begin by reviewing the classification of topological defects by looking at point defects in twodimensional nematics and line defects in three-dimensional nematics. In Sec. III we continue to point defects in threedimensional nematics. With all the characters introduced, we proceed to discuss our main point: that there are only two types of unlinked disclination loops. What we present is built on the classic review by Mermin (1979) on topological defects and a definitive result by Jänich (1987) that finds the topological classes of uniaxial nematic textures on $\mathbb{S}^{3}$, the three-dimensional sphere (equivalently, three-dimensional space with fixed boundary conditions at infinity), with point and line defects. We also discuss the biaxial nematic phase and show that it affords additional physical insight into the analysis of uniaxial defects.

\section{CIRCULAR LOOPS AROUND DEFECTS: THE FUNDAMENTAL GROUP}

A common and distinctive experimental procedure for imaging the orientational order is to place the sample between crossed polarizers, yielding in nematic materials the characteristic Schlieren texture from which they derived their name. In Fig. 1 our eyes are drawn to the dark brushes and tend to follow them toward their intersections. The brushes typically meet at points in twos or fours; larger numbers are possible but they too are multiples of two. The dark brushes correspond to regions where the orientation is parallel to either of the mutually perpendicular polarizer or of the analyzer directions and the points at which they meet are disclination defects. Why defect? Because there the orientation is ill defined, as the brushes tell us to assign two or more different orientations to that point. We can fix this by removing the defect point by poking a hole in the material. Physically, of course, we do not have a hole in our sample, just a place where we do not know the order. The problem is fixed by letting the magnitude of the order vanish at the origin. Then no hole is needed, but there is a point where there is no longer nematic order and the core is in the isotropic phase. To the reader acquainted with defects in superfluids and superconductors, this should sound familiar: In the center of an Abrikosov vortex in a conventional superconductor

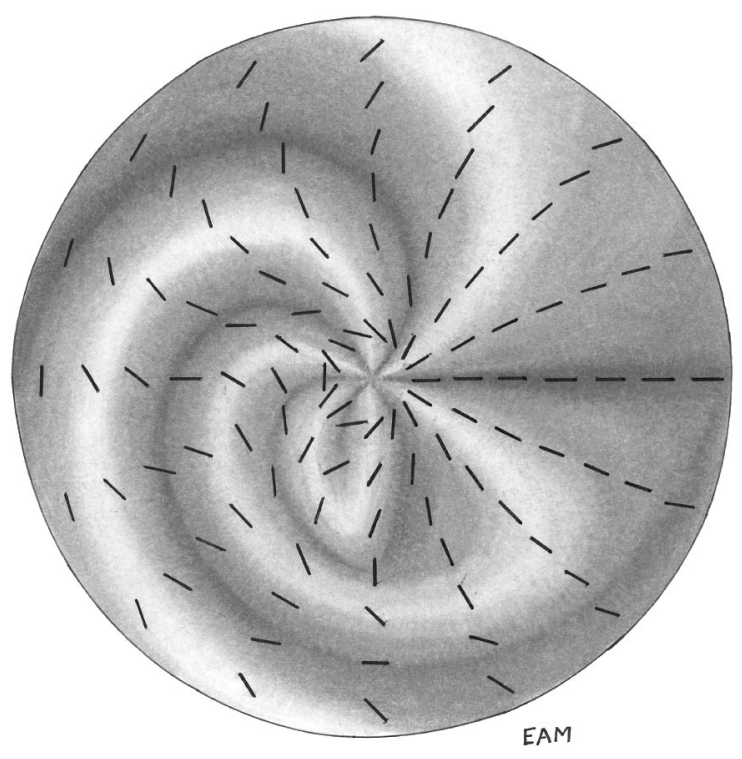

FIG. 1. Cartoon of the director field and the resulting brush pattern corresponding to the homotopy in Eq. (4). The radial coordinate $t$ parametrizes the sequence of maps $f_{t}(\phi)$. Near the defect center, six brushes are visible so that simply counting gives a winding of $\frac{3}{2}$. However, away from the defect core, the texture smoothly deforms to a texture that may be seen to have a winding of $\frac{1}{2}$. 
(Abrikosov, 1957) the superconducting order parameter vanishes and there is normal metal, a hole in the superconductor (Chaikin and Lubensky, 1995). In all these cases we can detect the existence of a defect without any detailed knowledge of the disordered core: Topological defects are characterized by the boundary conditions at the interface between the higher symmetry state and the lower symmetry state. In the Schlieren texture, these boundary conditions can be read off from the pattern of brushes.

In this section we introduce the basic concepts in the study of topological defects in this simplest case and connect the observation of brushes to the presence of defects. We cover what defects are, the use of measuring surfaces to probe them, and finally how to combine them. We begin by recalling that the continuum description of uniaxial nematics is based upon the assignment of a local "average molecular orientation" to every point in the sample, called the director field. This average orientation is that of a rodlike object, rather than an arrow, so that the nematic director is properly a line field, rather than a vector field. Moreover, it is only the orientation of the director that is important, not its magnitude, so that we can take the director to be a unit vector $\mathbf{n}$, subject to the condition that $\mathbf{n}$ and $-\mathbf{n}$ are identified.

\section{A. Two-dimensional director: $\mathbb{R} \mathbb{P}^{1}$}

First we consider nematic samples in which the director always lies in the $x-y$ plane, a prototypical thin cell situation. Equivalently, we consider the projection of a threedimensional director onto the $x-y$ plane:

$$
\mathbf{p}=A(x, y)[\cos \theta(x, y), \sin \theta(x, y)],
$$

where $A(x, y)$ is the nonvanishing magnitude of the projection and $\theta(x, y)$ is the angle it makes with the $\hat{x}$ axis. Just as $\mathbf{n}$ and $\mathbf{- n}$ are identified, so too are $\mathbf{p}$ and $-\mathbf{p}$ or, equivalently, $\theta(x, y)$ and $\theta(x, y)+\pi$. Because the polarizer and analyzer directions are $\pi / 2$ apart, each dark brush around a defect point tells us about a $\pi / 2$ rotation of the director. The number of brushes counts the winding of the director field. The fact that we have defects with two brushes directly tells us that rotations by $\pi$ leave the phase invariant and are experimental proof of the symmetry of the nematic phase (Frank, 1958). The angle that the director turns on a loop around a defect divided by the angle $2 \pi$ of a full turn is commonly referred to as the strength, or charge, of the disclination and is equal to $\pm 1 / 4$ times the number of brushes. Although the absolute value of the strength may easily be extracted from counting the number of brushes, the sign of the winding is not yet determined. It is a pleasant exercise in visualization to show that upon rotating the polarizers the brushes will counterrotate when the defect has negative winding and will corotate when the winding is positive.

As defects are points of discontinuity of the director field, we aim for a classification up to continuous deformations of their surroundings. When two paths can be continuously distorted into each other, they are said to be (freely) homotopic. It is useful to think of these deformations as time evolution because it not only allows us to visualize the distortion but it also reminds us that, under continuous dynamics, configurations remain in the same homotopy class. Above we began to think about a defect in terms of a measuring loop around it; this key idea is what allows us to probe the properties of a singularity in our texture without leaving the safety of the well-behaved director field. Consider the possibilities for the director on a small round measuring circle in the system. Here is an explicit family of possible measurements on this circle $\mathbf{p} / A=\mathbf{c}_{m}$ which exhibit any even number of brushes:

$$
\mathbf{c}_{m}=[\cos (m \phi), \sin (m \phi)]
$$

with $m \in \frac{1}{2} \mathbb{Z}$ a half-integer and $\phi$ the polar angle around our circle. These textures are all distinct in that we cannot continuously deform $\mathbf{c}_{n}$ into $\mathbf{c}_{m}$ for $n \neq m$. Thus within this family, the half-integer $m$ classifies the possibilities for the director field. Of course the textures described by Eq. (2) are special choices, or simplifications, and the director is rarely of this exact form even very close to the defect line. However, any director field that we might have measured on our circle can be homotoped into one of these. The utility of Eq. (2), therefore, is that these fields capture the possible windings of a general director field and thereby form a set of representatives for the behavior outside point defects. Most placements of our measuring circle will not surround a defect and hence the director field can be deformed to $\mathbf{c}_{0}$, which is a constant. On the other hand, if the measuring circle surrounds a point where two brushes meet, it should be homotopic to $\mathbf{c}_{1 / 2}$ or $\mathbf{c}_{-1 / 2}$.

Although reasonable sounding, the previously stated connection between brush counting and winding number relies on a hidden assumption. Suppose $\mathbf{p}=[\cos f(\phi), \sin f(\phi)]$ on our measuring circle and $f$ took the form

$$
f(\phi)= \begin{cases}\frac{3}{2} \phi & 0 \leq \phi<\frac{2 \pi}{3}, \\ 2 \pi-\frac{3}{2} \phi & \frac{2 \pi}{3} \leq \phi<\frac{4 \pi}{3}, \\ \frac{3}{2} \phi-2 \pi & \frac{4 \pi}{3} \leq \phi<2 \pi,\end{cases}
$$

where we first go from 0 to $\pi$, back to 0 , and then back to $\pi$. Now $f$ takes values in the interval $[0, \pi]$ with 0 and $\pi$ identified due to the $\mathbf{p} \rightarrow-\mathbf{p}$ symmetry. Were we to count the number of times we landed at $\pi / 2$, for instance, we would find 3 , as we would for any generic point of $[0, \pi]$. Under crossed polarizers we would see six brushes. But we can also smoothly distort the map $f(\phi)$ so that there are only two brushes through the sequence of maps $f_{t}(\phi), t \in[0,1]$ :

$$
f_{t}(\phi)= \begin{cases}\left(\frac{3}{2}-t\right) \phi & 0 \leq \phi<\frac{2 \pi}{3-2 t}, \\ 2 \pi-\left(\frac{3}{2}-t\right) \phi & \frac{2 \pi}{3-2 t} \leq \phi<\frac{(4-2 t) \pi}{3-2 t}, \\ \left(\frac{3}{2}-t\right) \phi+(2 t-2) \pi & \frac{(4-2 t) \pi}{3-2 t} \leq \phi<2 \pi .\end{cases}
$$

We see that $f_{0}(\phi)=f(\phi)$ while $f_{1}(\phi)$ generates the charge $1 / 2$ map $\mathbf{c}_{1 / 2}$ as $\phi$ ranges from 0 to $2 \pi$. So have we shown that charge $3 / 2$ is the same as charge $1 / 2$ ? Certainly not! Rather, we have learned that we must be more careful about counting brushes. We assumed in our previous rule of counting brushes to determine angles in which the angle function was always either increasing or decreasing on our loop. In 
general, when counting the number of times we pass $\pi / 2$ we must keep track of the way we pass it; is the angle increasing or decreasing? We thus look at the net rotation of $f(\phi)$ by calculating the winding number via the following integral:

$$
w=\frac{1}{2 \pi} \int_{0}^{2 \pi} d \phi \frac{d f_{t}}{d \phi}=\frac{1}{2},
$$

for all $t$. In general, the winding number is precisely the charge of the defect. In actual liquid crystal systems, energetics strongly disfavors extra brushes because these would correspond to regions of large splay or bend in a nematic. Thus, the naive counting of brushes works outside of exceptional circumstances. Although we framed the discussion above in terms of a particular measuring circle, the classification by winding numbers holds for any measuring loop disjoint from the defects, just let $\phi$ be any coordinate which goes from 0 to $2 \pi$ as we traverse the loop once. In particular, the texture on any loop in the sample that lassos just one of the defects is homotopic to any other; this can also be seen from the fact that the number of brushes meeting at the defect is conserved. Similarly, the number of brushes meeting at a point in a Schlieren texture remains unchanged in time as the texture evolves except when two defects combine and annihilate each other. The winding number $m$ is a key example of an essential idea in topology: turning geometric information into counting. By doing so we get robust measures since, in particular, integers (or half-integers), being discretely valued, cannot change continuously.

\section{B. Making precise measurements}

We start by putting our above classification into a broader context. We mentioned several different topological spaces that we should keep straight. First, we are dealing with uniaxial nematic textures, so there is a space associated with the possible ground states of the director field, the ground state manifold (GSM). ${ }^{1}$ Here the angle of the director $Y$ lives in the interval $[0, \pi]$ with 0 and $\pi$ identified. This space is known as the real projective line $\mathbb{R} \mathbb{P}^{1}$. As with any interval of the real line with end points identified, it has the topology of a circle. Next we have the space $M$ making up the sample volume. For our Schlieren textures, $M$ may be taken to be a suitable region of the plane. Inside $M$ there is a subspace, the set of defects $\Sigma$ where the order is not defined. In the last section, $\Sigma$ was the set of disclination points. We obviously have the director field, an $\mathbb{R} \mathbb{P}^{1}$-valued field on $M$ away from the defect set, $\mathbf{n}: M \backslash \Sigma \rightarrow$ GSM. In general, it is daunting to contemplate the texture on the entire sample especially if there are many defects. We also want to understand to what extent $\mathbf{n}$ may be understood as arising from its behavior near the defect set. Might there be a way to cut

\footnotetext{
${ }^{1}$ Recall that the ground states of a nematic are just uniform textures with the director pointing in some direction, so that in a general texture the local orientation can always be identified with one of these ground states. Thus the changing orientation of the director in a general texture may be thought of as motion on the ground state manifold. There is not an accepted term for this space in the literature, where it has also been called the manifold of internal states and the order parameter space.
}

$M \backslash \Sigma$ into more manageable pieces around its defects, classify those, and then glue them back together? These considerations motivate the introduction of additional, "auxiliary" spaces: measuring surfaces of fixed topology contained in the sample. We study the behavior of the director field on these as we vary their placement relative to the defect set or as we vary $\mathbf{n}$. Indeed, all the topological "charges" in this paper are defined with respect to a choice of measuring surface. Saying that a defect carries a charge is really shorthand for saying that a small measuring surface which surrounds only that defect measures such a charge. If such a measuring surface is not present, then the "charge of a defect" is ill defined. Of course, these ambiguities are nothing new and have been discussed before (Mermin, 1979). However, with the interest in nematics and cholesterics with embedded colloids (Muševič et al., 2006; Lintuvuori et al., 2010; Čopar and Žumer, 2011), these mathematical subtleties are no longer just about precision or axiomatic rigor; they are absolutely necessary for the proper interpretation of data. Above we measured the director on loops (which are topologically circles, denoted by $\mathbb{S}^{1}$ ) and observed that the winding number $m$ was constant during deformations of the loop or the texture as long as no defects pass through the loop, which essentially solved our classification problem for single defects in Schlieren textures. In more mathematical jargon, we saw that the set of maps from $\mathbb{S}^{1}$ to $\mathbb{R} \mathbb{P}^{1}$ up to homotopy (denoted $\left[\mathbb{S}^{1}, \mathbb{R P} \mathbb{P}^{1}\right]$ ) was equivalent to the set of half-integers $\frac{1}{2} \mathbb{Z}$.

How can we go from single defects back to the full texture? We draw a picture of a two-dimensional plane with punctures at the defects and small measuring loops about each puncture. Given the winding numbers at the loops, do we know the classification of the full texture? This picture is actually no different from one we might draw for the following situation: Consider a hypothetical Schlieren texture that contains several nearby strength $\pm 1 / 2$ defects. If we take a measurement around a circuit that surrounds all the defects, what do we get? Before we say anything more about the local-to-global question, we try to find an answer to this natural question: How can the theory capture the intuitive notion that defects can combine or split? We almost have it: We know that measuring circuits around defects are in correspondence with half-integers, and it happens to be true that any two half-integers can be added or subtracted. There is just a small gap between the question and answer now. For one, we need to define a way to go from two measuring circuits to one. The natural way to add loops is to concatenate them by forming a longer loop by running over the first and then running over the other. But in order to do this, the loops have to start and end at the same point in sample or they cannot be connected. By the same token, the two textures traced out on the loops must start and end at the same point in $\mathbb{R P}^{1}$. Given two arbitrary measuring loops, there is no reason for the textures on them to agree at some point. On the other hand, our figure shows that we can draw a big circle surrounding any pair. Is there a way to go from two circles to one without having to make arbitrary choices in the region between the circles? Yes. Since we are interested only in properties that are preserved under continuous deformations, we can deform the texture around one defect so that the value in $\mathbb{R} \mathbb{P}^{1}$ agrees at the common base point in the sample. To visualize this, consider a small 
disk around the defect. The homotopy proceeds radially outward by deforming the original texture at the center of the disk to the new texture at its outer edge.

This solution is inspired by the following geometrical fact: The space between a set of nonintersecting circles in the plane can be continuously deformed to a set of circles joined at a single point, called a wedge sum, or bouquet of circles as shown in Fig. 2. Once we have the texture on the bouquet, the concatenation of the loops is well defined, and we thus have a definition of addition in our system. In the case here, we can form addition by measuring around the loop which hugs the "outside" of the bouquet. By restricting to sets of loops which pass through the same base point, our set of equivalence classes of maps from $\mathbb{S}^{1}$ to the GSM is enriched with the additional algebraic structure of a group. Note that based homotopy is absolutely necessary here, continuous distortions of paths that preserve the base point.

A quick aside on group theory is in order. Recall that a group requires a way of adding its elements: We just saw that this is the concatenation of loops. In terms of brush counting, or the winding number, we need not be concerned with the details of the rate at which we traverse the GSM, or whether we pass a brush in the first or last half of the trip. We need only concern ourselves with the order in which we put the two paths together. This addition of classes of paths defines the group addition which may not be commutative but happens to be in this particular case. A group requires an identity: In a uniform nematic texture the map from a loop in the sample to the GSM is always constant, so this provides the identity under the group addition. Finally, each map has an inverse: Because we can go around the GSM in the opposite direction

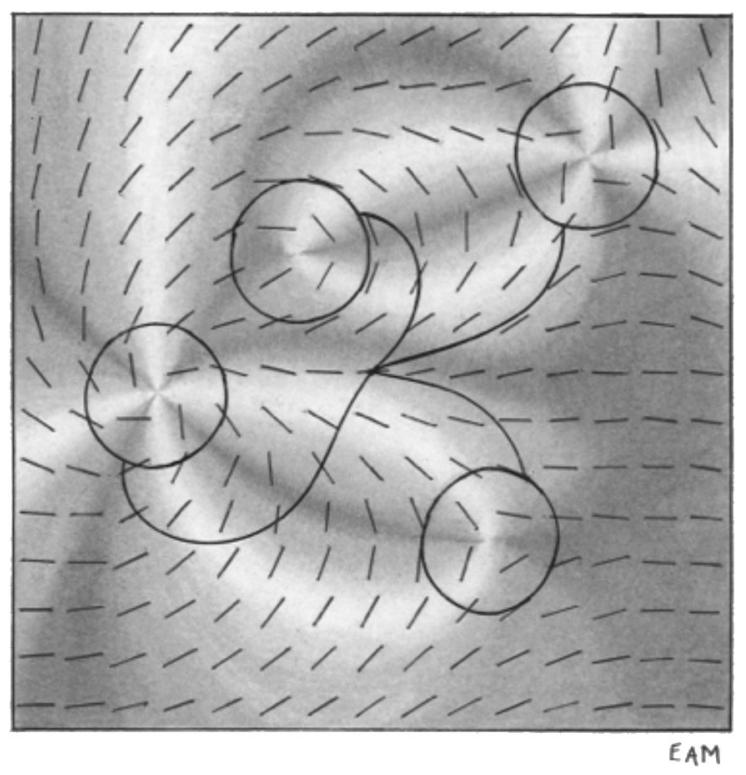

FIG. 2. A wedge sum or bouquet of circles. In order to make consistent measurements every measurement loop must be connected by a tether to a common base point in both the sample and the GSM. This way, measurements at different places can be joined together. Note that each tether is a path from the base point to the measurement loop and back, so that each of the four measuring circuits are homotopic to closed loops, encircling the appropriate defect, yet going through the common base point. by backtracking our precise path, we also have an inverse. Together these properties ensure that we have a group, known as the fundamental group, $\pi_{1}(\mathrm{GSM})$, the set of based maps from a measuring circuit $\mathbb{S}^{1}$ in the sample to the GSM which are equivalent up to based homotopy. And as the reader probably has already guessed, this group for the case where $\mathrm{GSM}=\mathbb{R} \mathbb{P}^{1}$ is precisely the (half-)integers. A loop that goes around only one defect in our hypothetical texture will intersect two brushes, giving a winding of $\pm 1 / 2$. A larger loop encircling both will yield a count of four if the defects have the same sign or zero if they have opposite signs. See Mermin's review (Mermin, 1979) for a more detailed and precise discussion of these properties.

Although the choice of the base point is arbitrary, its constancy is essential and leads to many of the interesting phenomena we discuss. The need for a base point is precisely why standard time zones were developed for trains. It is just a matter of having a single clock at one location (i.e., one measuring circuit) to determine the elapsed time at that spot. Two clocks can be used to measure elapsed time as long as they are synchronized, that is why we must have a base point. How are general maps from $\mathbb{S}^{1}$ to the GSM related to $\pi_{1}(\mathrm{GSM})$ ? In the case of Schlieren textures, we can relax: $\left[\mathbb{S}^{1}, \mathbb{R P} \mathbb{P}^{1}\right]$ is simply the underlying set of $\pi_{1}\left(\mathbb{R P} \mathbb{P}^{1}\right)$, that is, the set of maps without any addition rule. Even here, however, we must remember that if we want a topological charge for loops around defects that satisfies addition, we better pin our loops on a base point.

We conclude this section by sketching how what we have done tells us how to go from local measurements around defects to the full texture. The problem of classifying the defects on the full texture if we fix a base point is equivalent to that of classifying the texture on any bouquet of circles to which it may be retracted. ${ }^{2}$ For each circle in the bouquet, we can choose any element of $\pi_{1}\left(\mathbb{R P}^{1}\right)$, so the classification for a bouquet of $k$ circles is by a $k$ tuple of half-integers. In this case it turns out that this is also the answer if we allow the texture at the base point to vary. The complication, as we see in the following, is that there can be more than one way to tie together the measuring circuits. When $\pi_{1}(\mathrm{GSM})$ is nonAbelian this freedom of choice leads to an ambiguity in measuring the charge of a defect. This is an important message of this Colloquium and also emphasizes a possibly obvious point: The Abelian or non-Abelian nature of defects comes into play only when there is more than one defect. Making measurements correctly is, as always, the key to understanding the physical system.

\section{Three-dimensional director: $\mathbb{R} \mathbb{P}^{2}$}

In our two-dimensional example, we insisted that the director never point perpendicular to the plane and it was then possible to describe the texture in terms of idealized configurations where the director was entirely planar. This situation is often an accurate description of thin samples where the bounding surfaces are treated to promote planar

\footnotetext{
${ }^{2}$ It can be proved that "deformation retractions" of the sample onto a smaller space do not change the properties which can be probed with the tools of homotopy (Hatcher, 2002).
} 
alignment. However, in bulk samples, the nematic director can point along any of the directions in three dimensions with important implications for the topology.

The defects described in this section may still be captured by a measuring circle in the sample; in a three-dimensional nematic, these are line defects. By considering a twodimensional cross section of a three-dimensional nematic, we can discuss measuring circles around line defects as circles surrounding points in the plane, as in the previous section. Thus the sum of the measuring circles about two line defects corresponds to the process of merging two parallel defect segments into one. It is not hard to see that the constructions explained in the last section have direct analogs for line defects probed this way, and we leave elaboration on most of them to the reader. In later sections, we probe line defects with other measuring surfaces.

The GSM here is a sphere with antipodal points identified (as $\mathbf{n}$ and $\mathbf{- n}$ are identified in a line field). In this instance, the geometry is simple and we are able to see in our mind's eye the topology of the GSM. In more general situations, it is useful to have something more systematic: Indeed, without a systematic approach we can never be sure that our intuition is not fooling us. The general framework of the Landau theory of phase transitions that exploits symmetries and symmetry breaking not only provides well-known, systematic tools to our problem, but also emphasizes the essential aspect of topological defects: a higher symmetry phase surrounded by a lower symmetry phase.

Recall that any rotation in three dimensions is an element of $\mathrm{SO}(3)$, and one set of coordinates on this space consists of the three Euler angles. We write the rotation matrices as $\mathbf{R}_{\alpha \beta \gamma}=\mathbf{N}_{\alpha} \mathbf{M}_{\beta} \mathbf{N}_{\gamma}$, where the matrices $\mathbf{M}_{\beta}$ and $\mathbf{N}_{\gamma}$ are

$$
\begin{aligned}
\mathbf{M}_{\beta}= & {\left[\begin{array}{ccc}
\cos \beta & 0 & \sin \beta \\
0 & 1 & 0 \\
-\sin \beta & 0 & \cos \beta
\end{array}\right], } \\
\mathbf{N}_{\gamma}= & {\left[\begin{array}{ccc}
\cos \gamma & \sin \gamma & 0 \\
-\sin \gamma & \cos \gamma & 0 \\
0 & 0 & 1
\end{array}\right], }
\end{aligned}
$$

and $\alpha, \gamma \in[0,2 \pi)$, and $\beta \in[0, \pi]$. Away from the defect, the director is a rotation of some fiducial direction, say $\hat{\mathbf{z}}$, so at each point $\vec{x}$ in space there are three angles $\alpha(\vec{x}), \beta(\vec{x})$, and $\gamma(\vec{x})$, and $\mathbf{n}=\mathbf{R}_{\alpha \beta \gamma} \hat{\mathbf{z}}$. The uniaxial nematic state has two symmetries. First, because it is uniaxial, rotations around $\mathbf{n}$ leave the system invariant (a phase made of cylinders) and so we must identify $\mathbf{R}_{\alpha \beta \gamma}$ with $\mathbf{R}_{\alpha \beta \gamma^{\prime}}$ so that a rotation around the original $\hat{z}$ axis does not change the state. Since we may as well set $\gamma^{\prime}=0$, we first see that we can parametrize all directors by only two angles and we only need to consider the subspace of $\mathrm{SO}(3)$ represented by $\mathbf{T}_{\alpha \beta}=\mathbf{R}_{\alpha \beta 0}$. The group of rotations around a single axis is called $\mathrm{SO}(2)$ and this subspace made by $\mathbf{T}_{\alpha \beta}$ is called $\mathrm{SO}(3) / \mathrm{SO}(2)$, elements of $\mathrm{SO}(3)$ which are identified with each other by $\mathrm{SO}(2)$ rotations. Observe that this is a sphere, the space of configurations of a unit vector, appropriate for a spin in a Heisenberg magnet, and $\alpha$ and $\beta$ run over the standard azimuthal and polar angles on the sphere, respectively. In a nematic, $\mathbf{n}$ and $-\mathbf{n}$ are further identified; they are related by a rotation by $\pi$ around any axis perpendicular to the director $\mathbf{n}$. Again, we can prerotate the $\hat{\mathbf{z}}$ direction by $\pi$ using the diagonal matrix $\mathbf{P}=\operatorname{diag}[1,-1,-1]$, an element of $\mathrm{SO}(3)$ with $\mathbf{P}^{2}=\mathbf{1}$. The two element group made of $\mathbf{1}$ and $\mathbf{P}$ is called $\mathbb{Z}_{2}$. The nematic symmetry forces us to identify the two elements $\mathbf{T}_{\alpha \beta}$ and $\mathbf{T}_{\alpha \beta} \mathbf{P}=\mathbf{T}_{\alpha+\pi, \pi-\beta}$. The resulting space is $\mathrm{SO}(3) / H$, where $H$ is the group of nematic symmetries (the isotropy subgroup) which includes both $\mathrm{SO}(2)$ and $\mathbb{Z}_{2}$. Again, the notation $\mathrm{SO}(3) / H$ indicates that we identify two elements of $\mathrm{SO}(3)$, $\mathbf{R}$ and $\mathbf{R}^{\prime}$, if they differ by any element of the group made of $H=\left\{\mathbf{N}_{\alpha}, \mathbf{P} \mathbf{N}_{\alpha}\right\}$ for all $\alpha$. Note that $\mathbf{P} \mathbf{N}_{\alpha} \mathbf{P}=\mathbf{N}_{-\alpha}$ and so the product of two elements of $H, \mathbf{P}^{m_{1}} \mathbf{N}_{\alpha_{1}}$ and $\mathbf{P}^{m_{2}} \mathbf{N}_{\alpha_{2}}$, is

$$
\mathbf{P}^{m_{1}} \mathbf{N}_{\alpha_{1}} \mathbf{P}^{m_{2}} \mathbf{N}_{\alpha_{2}}=\mathbf{P}^{m_{1}+m_{2}} \mathbf{N}_{\alpha_{2}+(-1)^{m_{2}} \alpha_{1}} .
$$

While the total number of flips just adds $\left(m_{1}+m_{2}\right)$, the rotation angles do not; whether they are added or subtracted depends on $m_{2}$. Thus the group addition in $H$ is not just the group addition of the two different parts $\mathbb{Z}_{2}$ and $\mathrm{SO}(2)$ separately for $m$ and $\alpha$. $H$ is called the semidirect product of the two groups and, in this case, is the point symmetry group $D_{\infty}$, the proper symmetries of a right cylinder with a constant, circular cross section. In any event, the resulting space $\mathrm{SO}(3) / H$ has a name, the real projective plane $\mathbb{R P}^{2}$, and the identification of the spherical angles $(\theta, \phi) \leftrightarrow$ $(\pi-\theta, \phi+\pi)$ allows us to view it, just as our intuition suggested, as the sphere with diametrically opposed points identified, as shown in Fig. 3. However, the way that the angles add Eq. (7) may not have occurred to the casual observer without this analysis. ${ }^{3}$

This analysis demonstrates that based measuring circuits with the topology of a circle in a three-dimensional nematic and the one-dimensional defect structures they may encircle are characterized not by $\pi_{1}\left(\mathbb{R} \mathbb{P}^{1}\right)$ but rather by $\pi_{1}\left(\mathbb{R} \mathbb{P}^{2}\right)$, which turns out to be a much smaller group. Although when we traverse our measuring circuit in the sample the final orientation of the director field must be the same as the initial one, the final rotation $\mathbf{R}_{\alpha \beta \gamma}$ does not have to simply be the identity, it can be any symmetry transformation of the nematic, i.e., any element of $H=D_{\infty}$. This subgroup consists of two disconnected pieces, the rotations $\mathbf{N}_{\alpha}$ and composite rotations $\mathbf{P} \mathbf{N}_{\alpha}$. Homotopies can alter the precise value of the final rotation but not which connected component it is in. Consequently, we see that there are two classes of loops in $\mathbb{R} \mathbb{P}^{2}$. Returning to the geometric representation of $\mathbb{R} \mathbb{P}^{2}$ in Fig. 3 as the sphere with antipodal points identified, we can see that these two classes can be identified with loops that start and end at the same point on the sphere and those that start and end at antipodal points. The presence of a defect inside our measuring loop corresponds to the latter class of paths. In the previous case, we could simply count the number of brushes to determine that $\pi_{1}\left(\mathbb{R} \mathbb{P}^{1}\right)=\frac{1}{2} \mathbb{Z}$, the halfintegers. In the case of a true three-dimensional director we see that the group is actually simpler: $\pi_{1}\left(\mathbb{R P}^{2}\right)=\mathbb{Z}_{2}$ since

\footnotetext{
${ }^{3}$ Note that in our discussion of the two-dimensional nematic we could have described the GSM as $\mathbb{S}^{1}$ with diameters identified and gone through the same construction. The resulting space is $\mathbb{R} \mathbb{P}^{1}$, but its topology is identical to that of $\mathbb{S}^{1} . \mathbb{S}^{2}$ and $\mathbb{R P}^{2}$ are not topologically identical; in particular, they have different fundamental groups.
} 
(a)

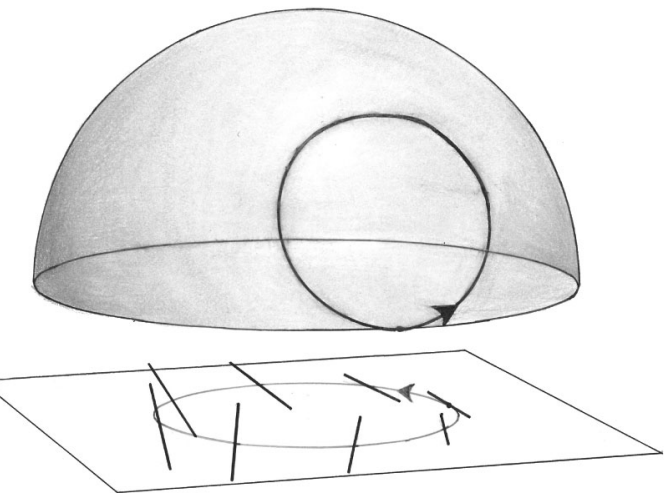

(b)

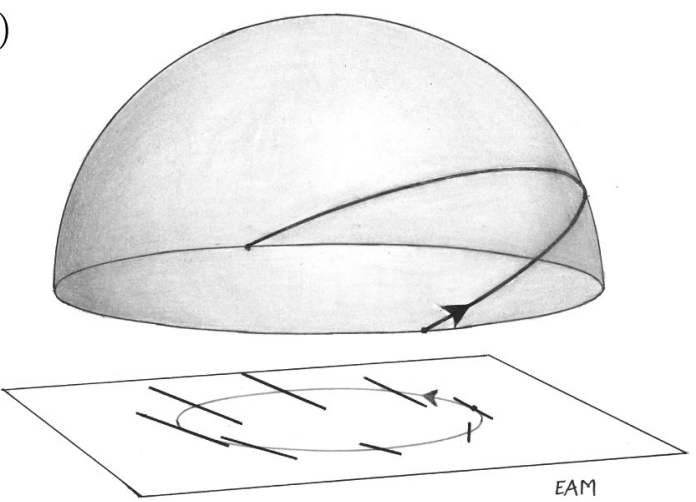

FIG. 3. The GSM for the three-dimensional nematic is the real projective plane $\mathbb{R P}^{2}$. Below each hemisphere which represents $\mathbb{R} \mathbb{P}^{2}$ we draw the path in the sample and the corresponding director at each point, with the arrows on the two curves denoting corresponding points and orientations. Any measurement around a closed loop in the sample can be mapped to a path in $\mathbb{R} \mathbb{P}^{2}$ which belongs to one of two homotopy classes: (a) trivial and (b) nontrivial. In other words, $\pi_{1}\left(\mathbb{R P} \mathbb{P}^{2}\right)=\mathbb{Z}_{2}$.

any closed path on $\mathbb{R} \mathbb{P}^{2}$ can be smoothly deformed (holding its base point fixed) either to its base point or to a path that connects antipodes. We write $\mathbb{Z}_{2}$ multiplicatively, so that it has the two elements $\{1,-1\}$. Thus, no distinction can be made any longer between positive and negative strength defects; the planar textures $\mathbf{c}_{1 / 2}$ and $\mathbf{c}_{-1 / 2}$ can be converted into each other by a uniform rotation of all of the molecules by $\pi$ about some axis in the plane, $\hat{x}$ say. On the GSM this rotation simply corresponds to transporting the loop $\mathbf{n}(\Gamma)$ from one side of the equator to the other by passing it over the North Pole, as shown in Fig. 4. Equally, there are no "winding 1" defects; the combination of circuits around two disclination lines in this case is always homotopic to a defectfree texture. For instance, the combination of the two planar textures $\mathbf{c}_{1 / 2}$ produces a loop on the GSM that goes all the way around the equator and so can be smoothly slid up in latitude until it shrinks to a point at the North Pole. ${ }^{4}$

\footnotetext{
${ }^{4}$ Strictly this particular homotopy does not preserve the base point, however, it is easy to construct one that does; simply hold that point of the loop fixed on the equator, slide the rest over the North Pole, and shrink it into the base point. In this case too, $\left[\mathbb{S}^{1}, \mathbb{R} \mathbb{P}^{2}\right]$ has the same elements as $\pi_{1}\left(\mathbb{R} \mathbb{P}^{2}\right)$. In general [ $\left.\mathbb{S}^{1}, \mathrm{GSM}\right]$ is equal to the underlying set of the group $\pi_{1}(\mathrm{GSM})$ whenever the latter is Abelian.
}
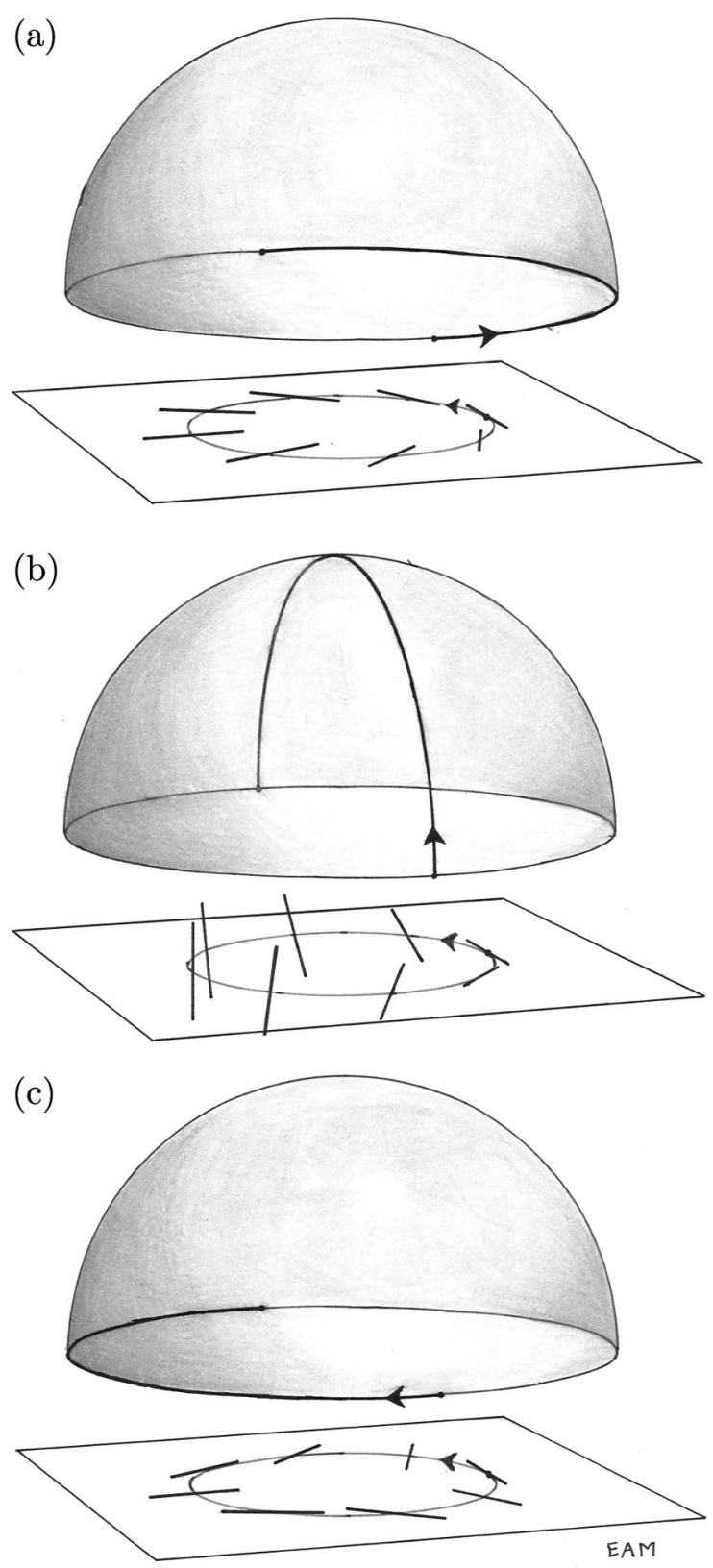

FIG. 4. The homotopy that takes the $a+\frac{1}{2}$ disclination line in a three-dimensional nematic into the $a-\frac{1}{2}$ disclination line consists of a uniform rotation of all the molecules by $\pi$ about the $\hat{x}$ axis. In $\mathbb{R} \mathbb{P}^{2}$ this homotopy entails sliding the path from the eastern half of the equator (a) over the North Pole (b) to the western half of the equator (c). As in Fig. 3 we draw the path in the sample and the corresponding director at each point below each picture of $\mathbb{R} \mathbb{P}^{2}$, with the arrows on the three curves denoting corresponding points and orientations.

The above homotopy from a loop covering the equator to a constant loop at the North Pole is actually observed in cylindrical capillaries treated to give perpendicular anchoring at the surface. The molecules rotate out of the plane so as to align along the cylinder axis in the interior, the so-called "escape in the third dimension" (Cladis and Kléman, 1972; Meyer, 1973; Williams and Bouligand, 1974). Of course, this escape can proceed in two directions: up or down. If, as typically happens, the molecules escape up in one section 
of the capillary and down in another then there will be a mismatch in between. This gives rise to a different type of defect: point defects in three dimensions, known colloquially in the field as hedgehogs (Polyakov, 1974).

\section{HEDGEHOGS}

Now that we have been introduced to the line defect, it behooves us to meet its partner, the hedgehog. Many features of the description of line defects carry over to the pointlike hedgehogs. Here we outline what is discussed in this section by sketching a few "three-dimensional versions" of the pictures described previously. First, a measuring loop can link with a defect line, but a loop cannot link with a point. Just as we need a Gaussian sphere to measure a point charge in electrostatics, we measure the topological charge of a point defect on a measuring sphere (denoted $\mathbb{S}^{2}$ ) in our sample. Thus what we seek to classify are the set of maps without base point $\left[S^{2}, \mathbb{R} \mathbb{P}^{2}\right]$ and the second homotopy group $\pi_{2}\left(\mathbb{R} \mathbb{P}^{2}\right)$. With that knowledge in hand, we consider a piece of the sample with multiple point defects by imagining a three-dimensional ball with measuring spheres around punctures at the hedgehogs. This space retracts to a bouquet of spheres, and the "sum" of the textures on the measuring spheres in the bouquet is taken to be the texture on a sphere that shrink wraps the entire thing. Just as for line defects, this picture relates a defect addition and a local-to-global construction.

We first study the group structure on $\pi_{2}(\mathrm{GSM})$, consisting of homotopy classes of based maps from the sphere to the GSM. Here, again, we fix a base point on both the sphere (which corresponds to the base point in our sample) and the GSM to define a group addition property. To be precise, consider the standard latitude and longitude coordinates on the globe. We choose the North Pole for the standard point on the sphere and some convenient point, say $Y_{0}$, in the GSM. To combine elements we imagine the following sequence of homotopies, depicted in Fig. 5. Consider first a single element $g_{1} \in \pi_{2}(\mathrm{GSM})$. We can smoothly deform the map by combing out a neighborhood of the North Pole into a polar cap which maps entirely to $Y_{0}$. We can keep smoothly deforming the whole map into a smaller and smaller patch of the sphere, until we have a small island surrounded by an ocean of points all mapping to $Y_{0}$. The texture arising on the island from a standard +1 hedgehog is a Skyrmion (Rößler, Bogdanov, and Pfleiderer, 2006; Mühlbauer et al., 2009) and the director rotates from $Y_{0}$ at the coast all the way to the antipodal direction $\bar{Y}_{0}$ in the interior, covering every orientation in between. This new map is homotopic to the original map which corresponded to $g_{1}$ and so, from the point of view of $\pi_{2}(\mathrm{GSM})$, the new map is the same element $g_{1}$. Remember the rate at which we traverse the GSM is not important, only the places visited. We can make the same smooth maneuver on a second element $g_{2} \in \pi_{2}(\mathrm{GSM})$, making a second island in the same ocean. To combine the two elements, we simply put island one $\left(g_{1}\right)$ and island two $\left(g_{2}\right)$ in the same ocean on the same sphere without overlapping the islands. This works because the boundaries of these islands are all mapped to $Y_{0}$. We can now smoothly deform this new map as we see fit, filling the ocean back up with land. In doing so we have combined the two elements. Since we can move the islands around before filling in the ocean, there is no way to order the group addition into $\pi_{2}(\mathrm{GSM})$ and it follows that $\pi_{2}$ is necessarily Abelian. As in the case of $\pi_{1}$, the identity element is again the equivalence class of the uniform texture that maps the whole sphere to $Y_{0}$.

Finally, the inverse of any element can be found by deforming the original map into an island and then taking the mirror image of the island through the sphere as in Fig. 6. Why does this give an inverse? We connect each point on the Western hemisphere to its reflection through the plane including the prime meridian in the Eastern hemisphere. We choose the value of the map on each of these lines inside the two sphere to take the value at the end points, identical by construction. It follows that we can fill the region inside the sphere with a smooth texture and there can be no defects inside. Thus the island and its mirror add to zero, shown in Fig. 6. Alternatively, we can take the map $f(\theta, \phi) \mapsto$ GSM and create the inverse $g(\theta, \phi)=f(\theta,-\phi)$.

We now relate the group structure of $\pi_{2}(\mathrm{GSM})$ to the addition of defects in terms of measuring surfaces: Imagine (as in Fig. 5) that we measure two different regions, each with a sphere. As for loops, in order to add the sphere measurements, they must be in a bouquet configuration. We can achieve this by attaching a tubelike tether to each sphere ending at our base point, but beware. The charges assigned to hedgehogs (actually, assigned to measuring spheres) will depend on the choice of tethers, particularly if there are disclinations in the system. The sum of the textures on these spheres is equivalent to the texture on a large sphere which has been deformed so that it snugly wraps around all of the original spheres. We encourage readers to relate this to the earlier picture of islands on a single sphere; each sphere in the bouquet becomes an island, and the base point of the bouquet is blown up to form the ocean. If no disclination lines are allowed, i.e., $\pi_{1}(\mathrm{GSM})=\{1\}$, then the tethers can always be unentangled since you "cannot lasso a basketball" (Coleman, 1988).

Although we have a definition and interpretation of $\pi_{2}\left(\mathbb{R P}^{2}\right)$, we have not computed it yet. In order to do so, we generalize the idea of counting brushes in the twodimensional nematic. Each brush is a place where the director points in one of two directions, parallel to the polarizer or analyzer, and the charge is one-fourth the number of brushes. Because of the cross polarizers, we are forced to count both the polarizer and analyzer directions. However, were we given an explicit map we could, instead, just count the number of times the map from $\mathbb{S}^{1}$ went to any single element of $\mathbb{R P}^{1}$ [with sign, as in Eq. (11)], an integer known as the degree of the map. Then the charge of the defect would be half the degree. We could try to do the same thing for maps from $\mathbb{S}^{2}$ to $\mathbb{R} \mathbb{P}^{2}$, but the ambiguity of $\mathbf{n}$ and $-\mathbf{n}$ makes this problematic. ${ }^{5}$ Each point of $\mathbb{R P}^{2}$ can be identified with two antipodal points on $\mathbb{S}^{2}$ : We start at the base point $Y_{0}$ and choose one of the two equivalent points on $\mathbb{S}^{2}$. Given a choice, we do this for all points in a small neighborhood

\footnotetext{
${ }^{5}$ The reader may worry that the same problem plagues us for $\pi_{1}\left(\mathbb{R} \mathbb{P}^{1}\right)$. It does not: $\mathbb{R} \mathbb{P}^{1}$ is orientable where $\mathbb{R} \mathbb{P}^{2}$ is not, which is the real issue here.
} 
(a)
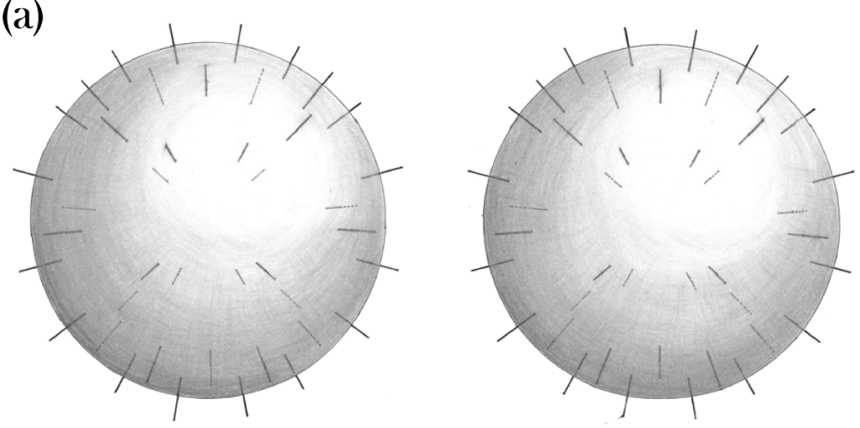

(c)
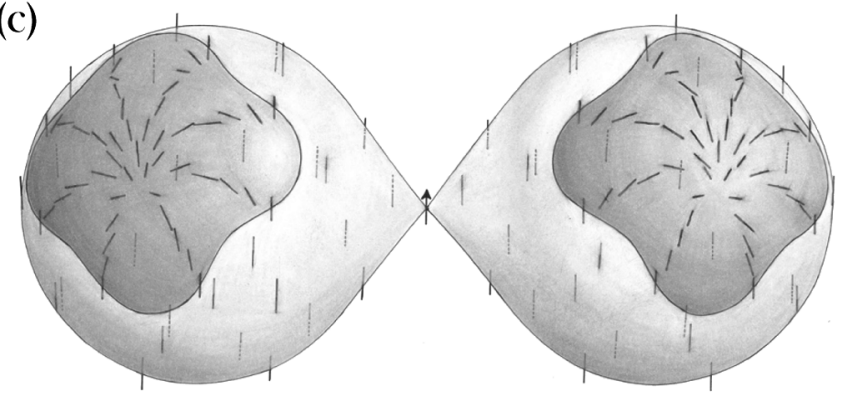

(b)

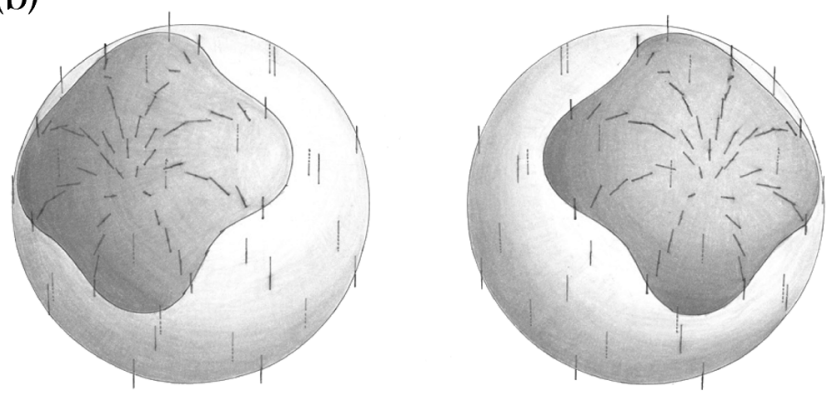

(d)

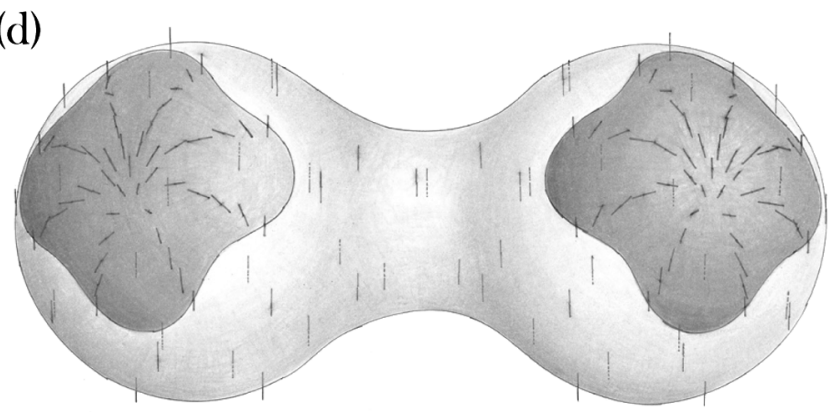

(e)

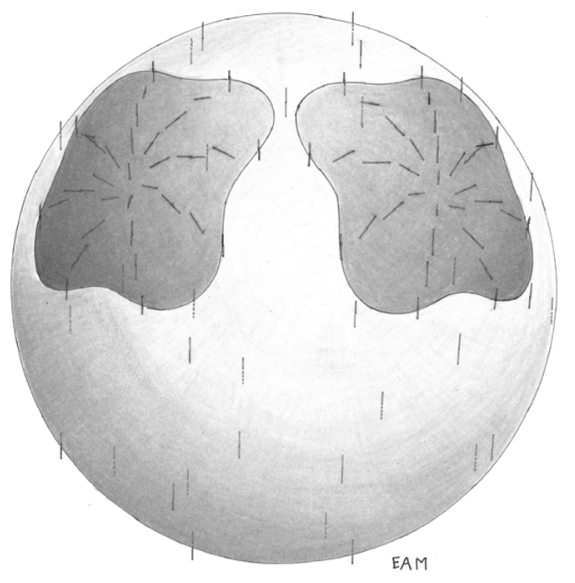

FIG. 5. How do you combine two hedgehog charges (a)? The key lies in based homotopy theory. First we comb the texture on the measuring surface so that the defect is confined to a small island within an ocean of uniform director field, which we identify as the base point (b). In order to compare the relative charges of two hedgehogs, their respective measuring surfaces must originate from the same base point, forming the bouquet of spheres described in the text (c). A new measuring surface, a sphere which has been shrink-wrapped around the bouquet of spheres, now contains both islands (d) and may be deformed into a single sphere containing the sum of both hedgehogs (e).

consistently so that we locally generate a new smooth map to $\mathbb{S}^{2}$. The only hitch might be that when taking a long path around $\mathbb{R P}^{2}$ we may find that we are hung up on a noncontractible path in the GSM when we return to the same point. ${ }^{6}$ However, this is impossible with maps from $\mathbb{S}^{2}$. Fortunately, maps from $\mathbb{S}^{2}$ to any space $X$ have a special property owing to the topology of the sphere. Consider a closed loop $\Gamma$ on $\mathbb{S}^{2}$ and the loop it produces on $X$ and watch how the latter changes as we smoothly deform $\Gamma$. Because $\Gamma$ can be shrunk to a point on $\mathbb{S}^{2}$, its image on $X$ can also be shrunk to a point and to one value in $X$. For the case of $\mathbb{R} \mathbb{P}^{2}$ this is convenient: The image of any loop $\Gamma$ on $\mathbb{S}^{2}$ must be

\footnotetext{
${ }^{6}$ This is the distinction between the identity and -1 in $\pi_{1}\left(\mathbb{R P}^{2}\right)$ : When paths in $\mathbb{R P}^{2}$ are lifted to paths in $\mathbb{S}^{2}$, paths in the equivalence class of the identity are closed, and paths in the class -1 are not.
}

homotopic to the identity map on $\mathbb{R} \mathbb{P}^{2}$, so we never get hung up. Once again, we cannot lasso a basketball and so we can always "lift" the map from $\mathbb{R} \mathbb{P}^{2}$ to $\mathbb{S}^{2}$ globally and turn it into a map from $\mathbb{S}^{2}$ to $\mathbb{S}^{2}$. Therefore, after fixing the lift of $\Upsilon_{0}$ (for definitiveness, we take it to lie in the Northern hemisphere), $\pi_{2}\left(\mathbb{R} \mathbb{P}^{2}\right)$ is the same as $\pi_{2}\left(\mathbb{S}^{2}\right)$, which we can calculate.

Each map gives us a unit vector $\mathbf{n}(\theta, \phi)$ at each point of the measuring sphere. Similar to the winding number, there is an integral that measures the number of times our map wraps around the sphere, also known as the degree or hedgehog charge

$$
d=\frac{1}{4 \pi} \int_{\mathbb{S}^{2}} d \theta d \phi \mathbf{n} \cdot\left[\partial_{\theta} \mathbf{n} \times \partial_{\phi} \mathbf{n}\right]
$$

Remarkably, it is an integer: The integrand is just the Jacobian of the map from $\mathbb{S}^{2}$ to $\mathbb{S}^{2}$ and so it counts the 


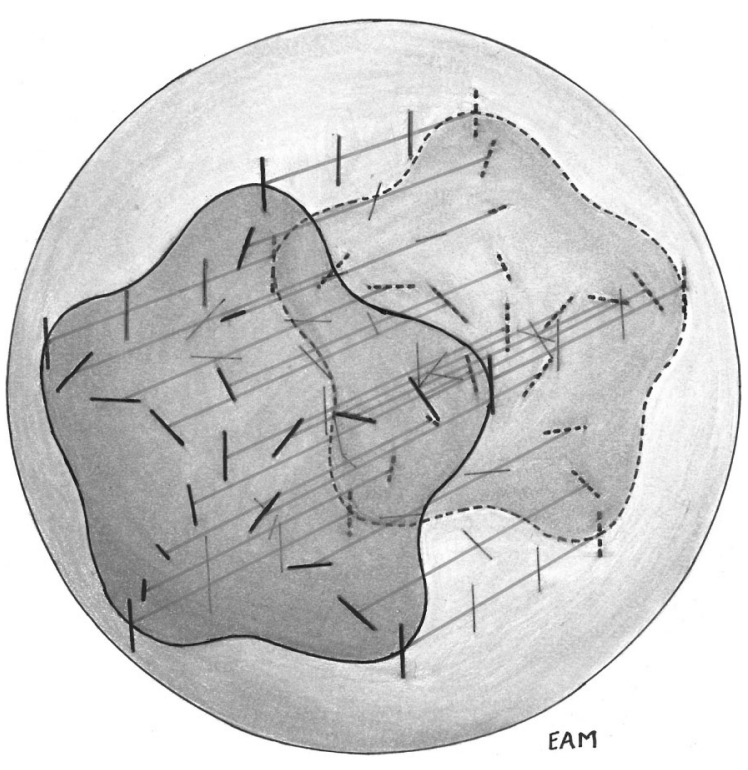

FIG. 6. The inverse of an element consists of taking the mirror image of its island and placing this new island on the sphere. The sum of these two elements must add so that the sphere contains no defects. In the interior of the sphere, the texture is constant along chords connecting identical points on the original island and its mirror. Because no defects are introduced, the entire texture on the sphere may now be smoothly deformed to the base point.

area swept out on the target sphere. Dividing by $4 \pi$ simply gives us the number of times we visit each point on the target. Moreover, because we do not take the absolute value of the Jacobian, we measure positive and negative area and thus will properly count the analog of increasing and decreasing as needed in Eq. (5) for the winding number. See, for instance, Kamien (2002) for the derivation of this Jacobian and its connection to Gaussian curvature. Therefore, we see that maps from $\mathbb{S}^{2}$ to $\mathbb{S}^{2}$ are classified by a degree and that this degree can be any integer, positive or negative. Thus $\pi_{2}\left(\mathbb{R P}^{2}\right)=\pi_{2}\left(\mathbb{S}^{2}\right)=\mathbb{Z}$, and there are an infinite number of topologically distinct point defects in nematic liquid crystals. Representatives of each homotopy class are given by the maps

$$
\mathbf{n}_{d}(\theta, \phi)=[\sin (\theta) \cos (d \phi), \sin (\theta) \sin (d \phi), \cos (\theta)],
$$

which exhibit a $d$-fold winding on the equator and may be thought of similar to the textures $\mathbf{c}_{m}$ of Eq. (2) as providing an idealized description of the director field near a hedgehog.

Just one point remains to straighten out: the question of the choice of lift. This will also tell us about the difference between the free homotopy classes, the set $\left[S^{2}, \mathbb{R} \mathbb{P}^{2}\right]$, and the second homotopy group $\pi_{2}\left(\mathbb{R P}^{2}\right)$. Suppose we had instead chosen to lift the base point $Y_{0}$ into the Southern hemisphere of $\mathbb{S}^{2}$. What would be different? We would still be able to identify each element of $\pi_{2}\left(\mathbb{R P}^{2}\right)$ with one in $\pi_{2}\left(\mathbb{S}^{2}\right)$ and define a degree $d$ through Eq. (8). However, when we would have used the vector $\mathbf{n}(\theta, \phi)$ to specify our texture we now use $-\mathbf{n}(\theta, \phi)$. Since Eq. (8) is odd in $\mathbf{n}$ the degree we now measure would be $-d$ instead of $d$. This is not to say that the textures labeled by $d$ and $-d$ are the same. The identification we made between $\pi_{2}\left(\mathbb{R P} \mathbb{P}^{2}\right)$ and $\pi_{2}\left(\mathbb{S}^{2}\right)$ is one to one; it is just that there are two identifications that we can make, differing in whether we lift the base point to the Northern or
Southern hemisphere, and these are not the same. ${ }^{7}$ So long as we make our choice of lift consistently, hedgehogs with charge $d$ and $-d$ are distinct, but it is up to us which sign we associate with which hedgehog. ${ }^{8}$

However, under free homotopy maps from the sphere to $\mathbb{R} \mathbb{P}^{2}$ labeled by $d$ and $-d$ do become equivalent. Take the texture of Eq. (9) and rotate uniformly by $\pi$ around the $\hat{y}$ axis, and then take $\mathbf{n}$ to $-\mathbf{n}$ to change $d$ to $-d$. It follows that $\left[\mathbb{S}^{2}, \mathbb{R} \mathbb{P}^{2}\right]$ is the set of non-negative integers, which is not the set of elements in $\pi_{2}\left(\mathbb{R P}^{2}\right)$. Thus even with the same spherical measuring surface, the classification of hedgehog defects can be different depending on how we make the lift from $\mathbb{R} \mathbb{P}^{2}$ and $\mathbb{S}^{2}$. As long as we do not try to extend the local measurements to a global texture, the "local-to-global problem," we need not be careful. However, if we specify free homotopy classes on spheres about defects and then attempt to fill the remaining space, we run into ambiguities or trouble. Were we to probe each hedgehog individually by calculating the degree on a small sphere around it, we would only be measuring its charge up to a sign, and we are assigning it to one of the maps $\left[\mathbb{S}^{2}, \mathbb{R} \mathbb{P}^{2}\right]$. The ease with which we do this extracts a penalty; we can no longer add defects since we have lost the group structure of $\pi_{2}\left(\mathbb{R} \mathbb{P}^{2}\right)$ without the use of a base point. For instance, a pair of spheres with $|d|=1$ on both might induce either $|d|=0$ or 2 . We cannot know the relative signs unless we have a common reference coming from a fixed base point.

To understand the global structure it is especially illustrative to view the sample as a bouquet of spheres attached at a single point, the base point of the sample, that maps to the base point of the GSM. If we have $k$ point defects then the texture becomes a map from a bouquet of $k$ spheres to $\mathbb{R} \mathbb{P}^{2}$. Were we to measure the charge on each sphere using the base points, we would end up with a $k$ tuple of elements of $\pi_{2}\left(\mathbb{R} \mathbb{P}^{2}\right)$ labeled by their degree $\left(d_{1}, d_{2}, \ldots, d_{k}\right)$. There is always a global ambiguity when we lift to $\mathbb{S}^{2}$ since the GSM base point can be taken to lie in either the Northern or Southern hemisphere. If we now choose to consider the free homotopy classes of samples with $k$ defects, we identify $\left(d_{1}, d_{2}, \ldots, d_{k}\right) \quad$ with $\quad\left(-d_{1},-d_{2}, \ldots,-d_{k}\right)$, not $\left( \pm d_{1}, \pm d_{2}, \ldots, \pm d_{k}\right)$. In other words, it is possible to go from a based measurement of a single defect to a based measurement of a collection of defects to an unbased measurement of many defects. However, it is not possible to make an unbased measurement of many defects from unbased measurements of single defects. This is the crux of the local-toglobal problem and is why local information is not always enough to understand the topology of the whole texture.

\footnotetext{
${ }^{7}$ The reader might be concerned that the same ambiguity arises for $\pi_{1}\left(\mathbb{R} \mathbb{P}^{1}\right)$. However the two choices of lift from $\mathbb{R} \mathbb{P}^{1}$ to $\mathbb{S}^{1}$ differ by a $\pi$ rotation, i.e., to replacing $f_{t}(\phi)$ with $f_{t}(\phi)+\pi$ in Eq. (5), which does not change the winding number $w$. In this case both lifts yield the same identification of $\pi_{1}\left(\mathbb{R} \mathbb{P}^{1}\right)$ with $\pi_{1}\left(\mathbb{S}^{1}\right)$.

${ }^{8}$ The prevailing convention in the literature is to consider the purely radial texture, Eq. (9) with $d=1$, to have positive charge, the choice of lift being that the director field points outward. The base point on the measuring sphere would be the North Pole and the base point in $\mathbb{R} \mathbb{P}^{2}$ is the vertical direction, which is then chosen to lift to the North Pole in $\mathbb{S}^{2}$.
} 


\section{DISCLINATION LOOPS}

We have now described disclination lines and hedgehogs separately, but in any typical texture both will be present simultaneously, and we are forced to think about how they interact with each other. When we discussed defect lines previously, we merely generalized point defects into a third direction and classified them by measuring circuits which ensnare the defect lines. However, these measurements could not determine whether or not the disclination itself is a loop or a knot, or linked with other disclinations. Indeed, part of the appeal of colloidal systems is precisely that they provide a natural setting for illustrating the topological interplay between disclinations and hedgehogs and enable the braiding and tangling of the disclination lines (Tkalec et al., 2011). If the colloid is treated to promote radial anchoring of the molecules at its surface then it will appear as a unit strength hedgehog and this topological charge will have to be compensated by a companion defect in the liquid crystal so as to satisfy the global boundary conditions. Another way of saying this is that the homotopy class of the texture on a measuring sphere which surrounds just the colloid differs from the one on a much larger measuring sphere on which, say, the texture is determined by the global boundary conditions. This implies the existence of another defect set. One way of achieving this balance is to place an opposite strength point defect close to the colloid so that together they form a dipole pair (Poulin et al., 1997). Multiple dipolar colloids interact with each other elastically through the distortions they produce in the liquid crystal and can as a consequence assemble into chains or two-dimensional colloidal crystals (Muševič et al., 2006; Škarabot et al., 2007).

A separate possibility is to have the charge of the colloid compensated by a disclination loop, encircling the particle in a Saturn ring configuration (Terentjev, 1995). These too interact elastically and aggregate to form chains and crystals (Škarabot et al., 2008). However, they also allow for a variety of intriguing entangled structures where a single disclination loop wraps around two or more colloids, balancing their collective charge (Guzmán et al., 2003; Ravnik et al., 2007; Ravnik and Žumer, 2009). This naturally begs the question of how to describe the topological properties of such disclination loops, which may be probed as both line and point defects. Although this makes sense from the perspective of conserving total charge, hedgehog charge, it turns out is not a homotopy invariant of a the full measuring surface of a disclination loop: a torus.

\section{A. Measuring with spheres}

Consider a based measuring sphere in our sample which does not surround any defect. The nematic texture can be deformed so that the sphere has one island with a +1 charge and a mirror island with charge -1 . As shown in Fig. 7, we can imagine distorting the sphere into a dumbbell shape with one island on each end. As we pinch off the tether connecting the two ends, each island comes to reside on its own sphere, $S_{+}$and $S_{-}$, surrounding a plus and minus hedgehog, respectively, which we realize were necessarily created during the process. Suppose that elsewhere in the sample there is a $1 / 2$ disclination line. What happens if we drag the -1 hedgehog around that disclination? More precisely, we keep the texture fixed on $S_{+}$so that the degree integral is unchanged but begin deforming $S_{-}$so that it wraps once around the disclination line and returns to its original position, leaving a tail tethered around the disclination line. Now the most natural sphere around the -1 hedgehog, $S_{-}^{\prime}$, is one that does not have the tether and is the one which we would likely use to measure the charge locally. What is the relation between the textures on $S_{-}$ and $S_{-}^{\prime}$ ? It can be difficult to visualize this operation, which involves a homotopy of a three-dimensional line field, but if we return to the original undeformed texture, the texture on $S_{-}^{\prime}$ is in fact equivalent to the one on $S_{-}$except that we then have attached a loop to it which goes around the disclination line in the "opposite direction" to the base point. Roughly speaking, $S_{-}$and $S_{-}^{\prime}$ clasp the hedgehog from different sides of the line defect. We call this set of operations maneuver $\mathbb{X}$. Such a tether picks up the $\pi$ rotation of the disclination and we are forced to change $\mathbf{n}$ to $-\mathbf{n}$ on most of the sphere, in particular, over the island, when we lift the texture on $S_{-}^{\prime}$ from $\mathbb{R} \mathbb{P}^{2}$ to $\mathbb{S}^{2}$. Thus when we calculate the degree on $S_{-}^{\prime}$ via integration, $d$ will go to $-d$. We have reversed the degree of the map on the sphere around the hedgehog by moving it around a disclination. Note that we had to carefully compare two distinct measuring spheres around the hedgehog in question in order to make sense of this. We started with total charge 0 around the two hedgehogs and ended with total charge \pm 2 , because we changed the measuring surfaces; just as the total charge in a Gaussian measuring box cannot change, neither can the charge in a fixed measuring circuit.

We can also interpret maneuver $\mathbb{X}$ from the point of view of "islands on the globe." Imagine a disclination loop that approaches the globe. We may poke an island on the sphere through this loop, and while performing this process the loop leaves an image in the shape of an atoll in the $Y_{0}$ ocean. Although the value in $\mathbb{R} \mathbb{P}^{2}$ can be the same inside and outside the atoll, when we lift to $\mathbb{S}^{2}$ we must change the sign of $\mathbf{n}$ when we cross the atoll, as this is equivalent to wrapping around the disclination loop. If the atoll is surrounding only a region of open ocean, this is not a problem. We can shrink it to a point and effectively make the whole region of the "wrong" ocean of $\bar{Y}_{0}$ disappear. But given an island carrying a nontrivial hedgehog charge, when we lift to $\mathbb{S}^{2}$ we will be forced to match the island's coastline to a lagoon of $\bar{Y}_{0}$. No problem, just lift the island to $-\mathbf{n}$ instead of $\mathbf{n}$. Is there a singularity of the texture on the atoll? No. For concreteness take $Y_{0}$ to be the North Pole and so $\bar{Y}_{0}$ is the South Pole. Topologically, the atoll is an annulus. The inner ring of the annulus points south and the outer ring points north. It is no problem to have the director smoothly rotate along the radius of the annulus from south to north. This texture will not contribute to the degree. By stretching the measuring surface this becomes precisely the picture in Fig. 7 where the atoll becomes the tubelike tether. Again, this socalled "action" of $\pi_{1}$ on $\pi_{2}$ preserved the island's class in the set of unbased maps $\left[\mathbb{S}^{2}, \mathbb{R}^{2}\right]$, but not in $\pi_{2}\left(\mathbb{R} \mathbb{P}^{2}\right)$.

\section{B. Measuring with tori}

Hedgehog charge is a homotopy invariant of a sphere, the natural measuring surface for a point defect. But the natural 
(a)
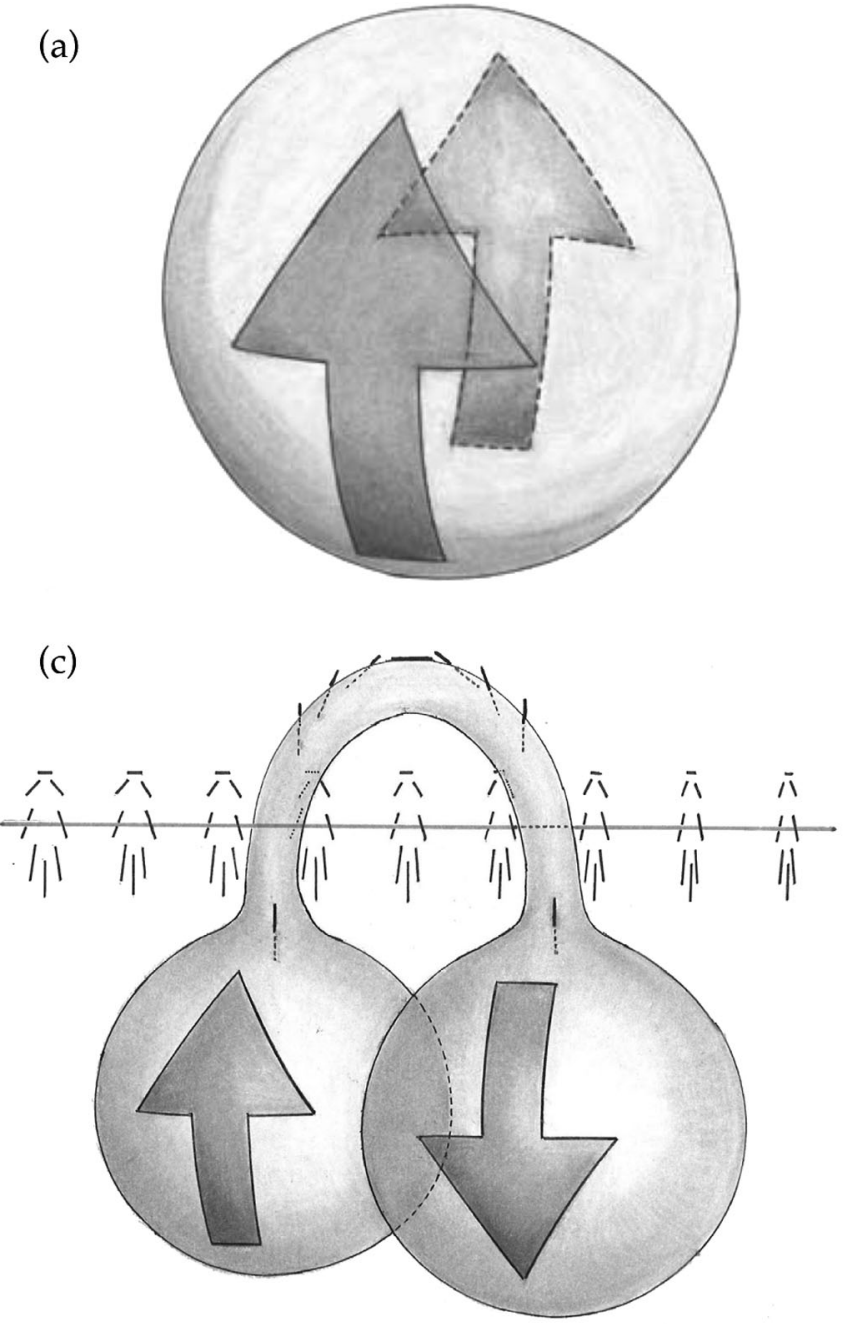

(b)

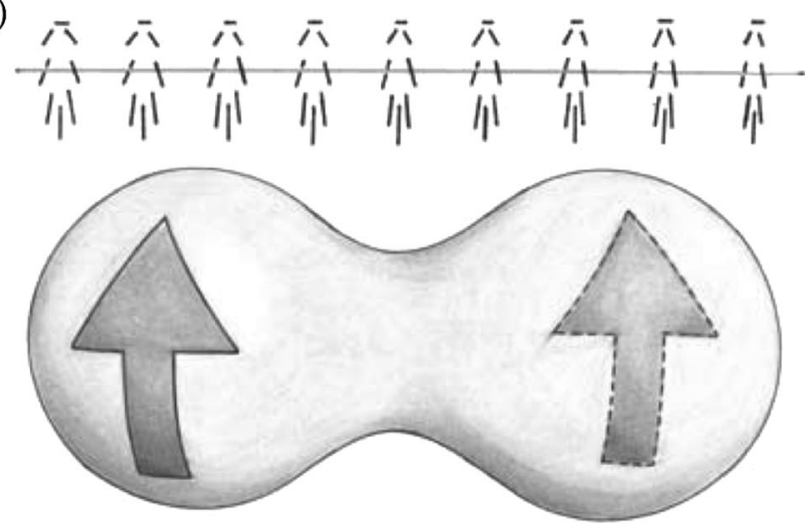

(d)

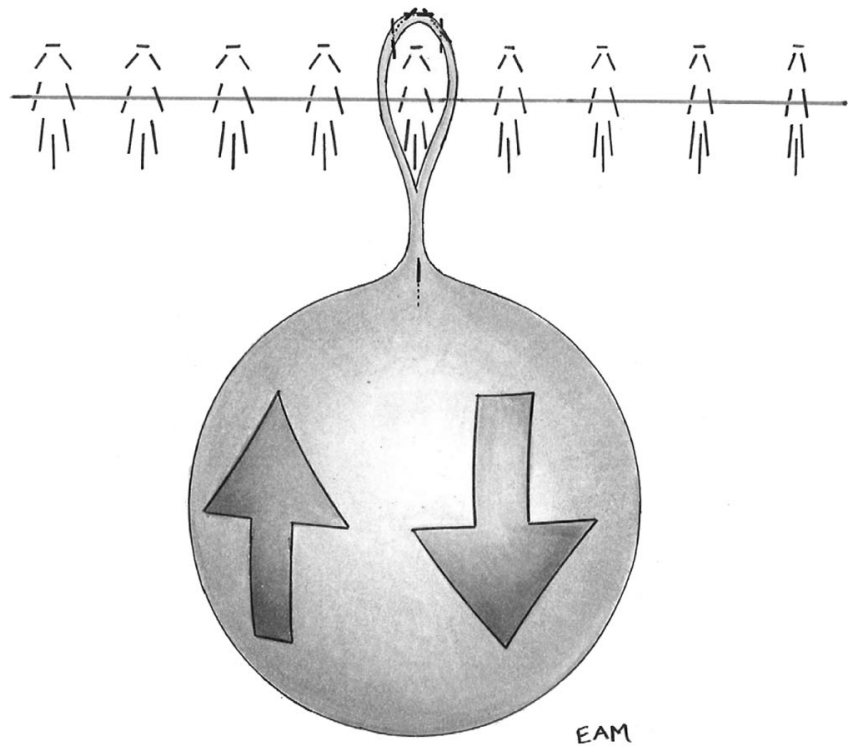

FIG. 7. (a) A sphere with a plus and minus island has zero net hedgehog charge. (b) The two islands can be separated to create two spherical bulges $S_{+}$and $S_{-}$for the plus and minus pair. If we pull one sphere around the disclination and insist on keeping the base point ocean fixed then the entire $\pi$ rotation of the director must occur in the space between the two spheres. This makes it impossible to bring the spheres together while deforming the nematic continously. Bringing the two oceans in contact would require an even more rapid twist in the nematic which would render the texture discontinuous. Therefore, it is necessary to have the $\pi$ rotation occur somewhere else if we want to join the spheres to create a single measuring surface. Thus there will be some region that can be pushed onto the tether where there is a $\pi$ twist. (c) This rotation of the ocean from "up" to "down" allows us to combine the spheres but changed the overall degree measured on one of the two spherical bulges, so as the minus hedgehog is carried around a disclination line its orientation reverses and the natural final measuring circuit ( $\left.S_{-}^{\prime}\right)$ records the opposite hedgehog charge. (d) If the two hedgehogs are now recombined and the tether is ignored, their total charge will be \pm 2 .

measuring surface for a disclination loop is not a sphere but a torus $\mathbb{T}^{2}$, a thin tube sheathing the singular line. The classification of disclination loops can therefore be based on the homotopy invariants of the texture on this torus, that is, on maps from $\mathbb{T}^{2}$ to $\mathbb{R} \mathbb{P}^{2}$. There are multiple measures of these maps, two corresponding to elements of $\pi_{1}\left(\mathbb{R} \mathbb{P}^{2}\right)$ measured on the cycles of the torus and a more refined quantity, a global defect index (Jänich, 1987; Nakanishi, Hayashi, and Mori, 1988; Bechluft-Sachs and Hien, 1999) which captures some aspect of hedgehog charge. A torus surrounding a simple, circular disclination loop, shown in Fig. 8, has two cycles: a meridional loop that goes around the disclination line which always measures the nontrivial element of $\pi_{1}\left(\mathbb{R} \mathbb{P}^{2}\right)$, and the loop that follows the contour of the disclination line along the longitude of the torus records another element of $\pi_{1}\left(\mathbb{R P}^{2}\right){ }^{9}$ If this is also nontrivial then it means that our disclination loop itself goes around another defect, linked loops, while if it is the trivial element then our disclination loop is either isolated or linked an even number of times. For now we

\footnotetext{
${ }^{9}$ The reader may worry about which longitudinal path to follow if the disclination loop has a more complicated shape or is knotted. A canonical choice is provided by any path that has zero linking number with the defect loop itself (Jänich, 1987).
} 


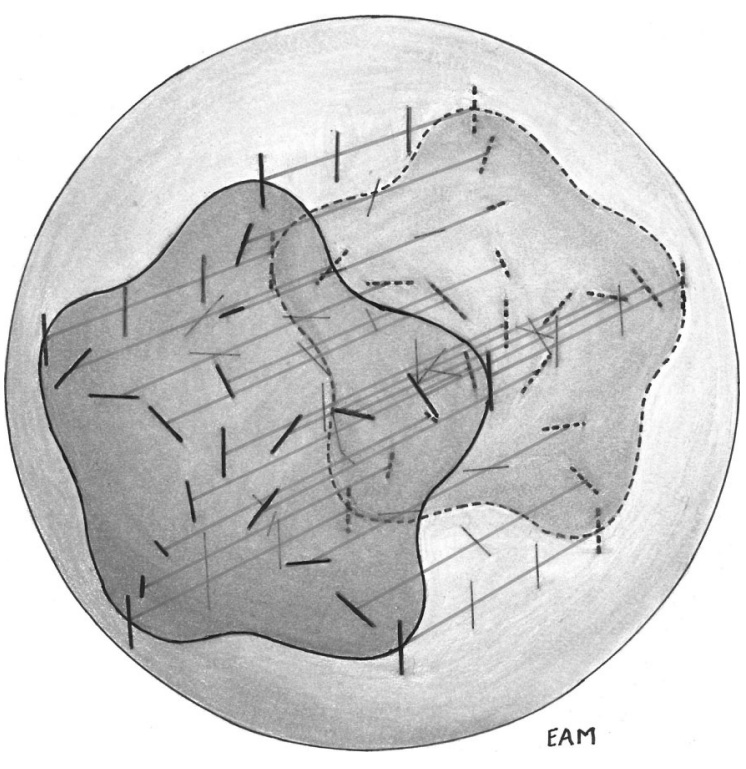

FIG. 8. The measuring surface $\mathbb{T}^{2}$ has two cycles, either of which may contain nontrivial winding. For the unlinked disclination loop shown the winding is nontrivial around the meridian and trivial around the equator.

assume that this element is trivial and that our disclination loop is unlinked.

What is the homotopy classification of this subset of textures on a torus? One naive guess would be that we would have an additional choice of integer $n$, which we might get by repeatedly merging hedgehogs into the line defect, in a way analogous to our way of merging two hedgehogs together (via a bouquet construction). However, we can show that adding two hedgehogs to our disclination is the same as not adding any by using maneuver $\mathbb{X}$, as in Fig. 9 . We have a texture on the torus called $f$; we now show that $f^{\prime}$ arising from adding two +1 islands to the torus by merging in a sphere that carries +2 hedgehog charge is homotopic to $f$. Move one of the +1 islands on $f^{\prime}$ on a loop around the meridian. As we pull the island around a circle of longitude it accumulates a concentric series of borders around the island which rotate the coastline by $\pi$. As before this is the action of the group element $-1 \in$ $\pi_{1}\left(\mathbb{R P} P^{2}\right)$ on the group element $+1 \in \pi_{2}\left(\mathbb{R} \mathbb{P}^{2}\right)$. This island is now homotopic to $-1 \in \pi_{2}\left(\mathbb{R} \mathbb{P}^{2}\right)$, as argued before. Now we have a \pm 1 pair of islands on the torus which we can cancel against each other just as we could for a pair of such islands on a sphere. This results in a texture on the torus which is homotopic to the one we started with. From another point of view, performing maneuver $\mathbb{X}$ does not require any discontinuous changes on the torus; the hedgehog moves through the handle without piercing the measuring surface. As a result, we can smoothly transform the texture on $\mathbb{T}^{2}$ while changing the hedgehog charge of the surrounding space by \pm 2 . Hence, we can classify tori surrounding disclination loops as carrying either even or odd hedgehog charge. The reader might ask how we can be sure that there are no moves that can change this charge by \pm 1 , an issue that we explain in the next section.

In what follows we state some interesting results that fill out most of the rest of the story about disclination loops. The complete classification of textures on tori labels textures with
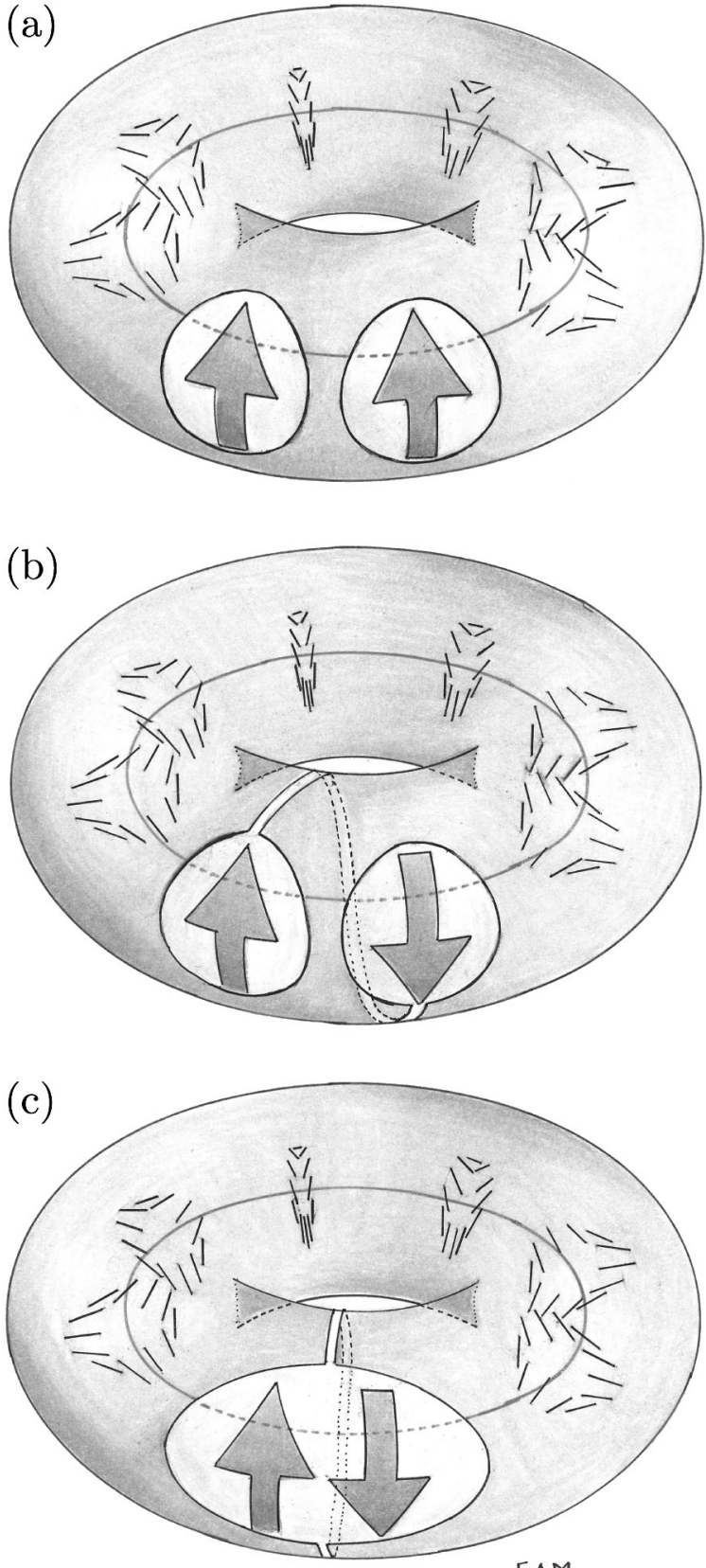

EAM

FIG. 9. (a) Two +1 islands on a torus surrounding a disclination loop, representing the addition of two hedgehogs. We can always tether the two islands together to measure the combined charge. (b) However, moving one of the islands around the meridian of the torus reverses its orientation, turning it into a charge -1 island. (c) If the two islands are then merged they carry zero net charge, illustrating the homotopy between disclination loops with hedgehog charge $n$ and $n \pm 2$. As in Fig. 7, there must be a $\pi$ rotation of the director somewhere on the tether in (b) and (c).

one of the four pairs $(a, b)$ where $a, b= \pm 1$ are the homotopy classes (of $\pi_{1}$ ) on the meridian and longitude cycles, respectively. For the three cases where $a$ and $b$ are not both +1 , it turns out there are two subclasses of textures within those that are labeled with $(a, b)$, which correspond to even and odd numbers of +1 islands on the torus, just as for the case $(-1,+1)$ we explained above. When $a$ and $b$ are both +1 , there are an infinite number of classes; without a 
TABLE I. A summary of classifications of textures on various measuring surfaces, their physical relevance, and how they may be combined. If free or based is not specified, the underlying sets for the classifications are the same.

\begin{tabular}{|c|c|c|c|}
\hline Physical system & GSM & Measuring circuit & Classification and notes \\
\hline Point defects in Schlieren texture & $\mathbb{R P}^{1}$ & $\mathbb{S}^{1}$ & $\frac{1}{2} \mathbb{Z}$, when based, can be added (see Sec. II.A). \\
\hline Disclination lines in $3 \mathrm{D}$ nematics & $\mathbb{R} \mathbb{P}^{2}$ & $\mathbb{S}^{1}$ & $\mathbb{Z}_{2}^{2}$, when based, can be multiplied (see Sec. II.C). \\
\hline Point defects in 3D nematics & $\mathbb{R} \mathbb{P}^{2}$ & $\mathbb{S}^{2}$ & $\begin{array}{c}\mathbb{N} \text { when free, } \mathbb{Z} \text { and may be added when based (see Sec. III). For } \\
\text { based textures, attaching a tether which runs over }-1 \in \\
\pi_{1}\left(\mathbb{R} \mathbb{P}^{2}\right) \text { takes } d \text { to }-d \text { (see Sec. IV). }\end{array}$ \\
\hline Disclination loops in 3D nematics & $\mathbb{R} \mathbb{P}^{2}$ & $\mathbb{T}^{2} \cong \mathbb{S}^{1} \times \mathbb{S}^{1}$ & $\begin{array}{l}(a, b)_{p}, \text { where } a, b= \pm 1 \text {, and } p=\text { even, odd if } a, b \text { are not both } \\
1 \text {, if } a=b=1 \text {, then } p \in \mathbb{Z} \text { if based, or } \mathbb{N} \text { if free (see Sec. IV). }\end{array}$ \\
\hline
\end{tabular}

disclination loop in the torus, we cannot cancel out pairs of islands anymore. ${ }^{10}$ We therefore can add a subscript in either $\{e, o\}$ (even or odd) or $\mathbb{Z}$ to this ordered pair to complete the classification.

This peculiar set of homotopy classes has some interesting additional structure: We may do much more with tori than just merge them with spheres, as we did above. We present below simply one example, which corresponds to merging two disclination loops (i.e., tori with $a=-1$ ) "side to side" (Jänich, 1987; Bechluft-Sachs and Hien, 1999). Unfortunately, a precise explication of the other group which emerges when we merge two unlinked tori (i.e., with $b=1$ ) "top to bottom" (Nakanishi, Hayashi, and Mori, 1988) is outside the scope of this paper. Given two tori with textures such that $a=-1$ on both, we cut them along a meridional circle, resulting in two cylinders and then reglue them so that we have a single torus. This results in a $\mathbb{Z}_{4}$ group structure on the homotopy classes of such textures on a torus, where the mapping is $[0]=(-1,1)_{e},[1]=(-1,-1)_{o},[2]=(-1,1)_{o}$, and $[3]=(-1,-1)_{e}$, where we write $\mathbb{Z}_{4}$ additively so that $[m]+[n]=[m+n \bmod 4]$. It is natural for $(-1,1)_{e}$ to be the identity element; it is both unlinked and may contain no hedgehogs, which makes adding it a bit like adding a "constant" segment of disclination line.

We summarize these and the results of the last three sections in Table I.

\section{BIAXIAL NEMATICS AND THE ODD HEDGEHOG}

In the last section, we argued that by a smooth deformation a map from $\mathbb{T}^{2}$ to $\mathbb{R} \mathbb{P}^{2}$ could absorb hedgehog charge in pairs so that the hedgehog charge could be only even or odd. Here we probe this further and use the insight provided by decorating the uniaxial textures with a small amount of biaxial order. This biaxial point of view both highlights the underlying topology of the uniaxial phase and clarifies the way in which the even and odd classes of uniaxial disclination loops are distinct.

Recall in our discussion of the nematic we discovered, via some matrix algebra, that the ground state manifold was $\mathbb{R P}^{2}$. This was appropriate for a uniaxial nematic, which had rotational symmetry around its long axis, the rotational symmetry

\footnotetext{
${ }^{10}$ Free and based homotopy classes have the same classification for the first three classes where $a, b$ are not both trivial, since multiplying by -1 does not change evenness or oddness of the hedgehog charge on the islands. For the $(+1,+1)$ case, the based classification is all integers, and the free classification is only non-negative integers, just as for textures on a sphere.
}

responsible for the $\mathrm{SO}(2)$ factor in $H$. A biaxial nematic has a lower symmetry, that of a brick or rectangular cuboid with three unequal lengths. Not just a mathematical construct, biaxial liquid crystalline phases have been known for many decades. In the past few years, discovery of thermotropic biaxial phases has renewed interest in their defect structures. Moreover, chirality and biaxiality are intimately connected (Priest and Lubensky, 1974; Harris, Kamien, and Lubensky, 1999), and studies of blue phases (Grebel, Hornreich, and Shtrikman, 1983; Wright and Mermin, 1989; Dupuis, Marenduzzo, and Yeomans, 2005) often utilize a biaxial description.

Returning to the notation and discussion in Sec. II.C, we start with the biaxial molecule with long axis along $\hat{z}$ and a second axis along $\hat{x}$ (the third axis is along $\hat{y}$ and all three are distinguishable: "triaxial nematic" might be more apt). The symmetry of the brick involves only three discrete rotations of $\pi$ around each of $\hat{x}, \hat{y}$, and $\hat{z}$. Ignoring those symmetries for the moment, we see that the original rotation matrices $\mathbf{R}_{\alpha \beta \gamma}$ represent an arbitrary rotation of the brick. With the symmetries, we must now identify $\gamma$ with $\gamma \pm \pi$, so that the isotropy subgroup $H_{b}$ becomes

$$
H_{b}=\left\{\mathbf{1}, \mathbf{P}, \mathbf{N}_{\pi}, \mathbf{P N}_{\pi}\right\}
$$

where the addition of elements, multiplication of the matrices in Eq. (6), is as before. Note that $\mathbf{P N}_{\pi}=\mathbf{M}_{\pi}$, so that a rotation around $\hat{z}$ of $\pi$ followed by a similar rotation about $\hat{x}$ yields a $\pi$ rotation around $\hat{y}$. For all three of these elements, we are never sure whether these rotations are by $\pi$ or $-\pi$. Of course, the reader might think that these lead to the same group elements, which they do. However, a loop in $\mathrm{SO}(3)$ that starts at 0 rotation and ends at a $2 \pi$ rotation is not contractible and leads to a nontrivial element of $\pi_{1}\left[\mathrm{SO}(3) / H_{b}\right]$. This fact, one might recall, is often demonstrated in a class on quantum mechanics by someone who takes off their belt or holds a filled coffee cup with one hand and performs an elegant gyration of their arm. ${ }^{11}$ It is why spinors must change sign under rotations by $2 \pi$ and why the spin and statistics of particles are interrelated. Now that we have introduced the notion of a lift when discussing the hedgehog charge, it is simplest to demonstrate this fact with yet another lift.

The Pauli matrices,

$$
\boldsymbol{\sigma}_{1}=\left[\begin{array}{ll}
0 & 1 \\
1 & 0
\end{array}\right], \quad \boldsymbol{\sigma}_{2}=\left[\begin{array}{cc}
0 & -i \\
i & 0
\end{array}\right], \quad \boldsymbol{\sigma}_{3}=\left[\begin{array}{cc}
1 & 0 \\
0 & -1
\end{array}\right]
$$

\footnotetext{
${ }^{11}$ Some readers may have even been subjected to "spinor spanners."
} 
satisfy $\boldsymbol{\sigma}_{i} \boldsymbol{\sigma}_{j}=i \epsilon_{i j k} \boldsymbol{\sigma}_{k}+\delta_{i j} \mathbf{1}$ as follows from their commutators and anticommutators. This can be used to form a simple way to parametrize rotations in three dimensions. Write any vector $\vec{x}=\left[x_{1}, x_{2}, x_{3}\right]$ as the matrix $\mathbf{x}=x_{k} \boldsymbol{\sigma}_{k}$ where we employ the summation convention over repeated indices. Then $\mathbf{x}^{2}=|\vec{x}|^{2} \mathbf{1}$ is the unit matrix times the squared length of the vector. Moreover, if $\mathbf{U}$ is any $2 \times 2$ unitary matrix, then if we define the similar matrix $\mathbf{x}^{\prime}=\mathbf{U}^{\dagger} \mathbf{x} \mathbf{U}$, we find that $\left(\mathbf{x}^{\prime}\right)^{2}=\mathbf{U}^{\dagger}|\vec{x}|^{2} \mathbf{1} \mathbf{U}=\mathbf{x}^{2}$, so under a unitary transformation the magnitude of the matrix is unchanged, precisely what we need for rotations. We finally note that for small $\vec{\theta}$, the matrix $\mathbf{U}(\vec{\theta})=\mathbf{1}-i \frac{1}{2} \theta_{k} \boldsymbol{\sigma}_{k}$ generates infinitesimal rotations of $\mathbf{x}$ since to linear order in $\vec{\theta}$ (note that these angles are not the same as the Euler angles $\alpha, \beta$, and $\gamma$ ):

$$
\begin{aligned}
\mathbf{U}^{\dagger} \mathbf{x} \mathbf{U} & =\left[\mathbf{1}+i \frac{1}{2} \theta_{k} \boldsymbol{\sigma}_{k}\right] x_{i} \boldsymbol{\sigma}_{i}\left[\mathbf{1}-i \frac{1}{2} \theta_{j} \boldsymbol{\sigma}_{j}\right] \\
& =\mathbf{x}+\frac{i x_{i} \theta_{k} \boldsymbol{\sigma}_{k} \boldsymbol{\sigma}_{i}-i x_{i} \theta_{k} \boldsymbol{\sigma}_{i} \boldsymbol{\sigma}_{k}}{2}+\mathcal{O}\left(\theta^{2}\right) \\
& =\left[x_{j}+x_{i} \theta_{k} \epsilon_{i k j}\right] \boldsymbol{\sigma}_{j}+\mathcal{O}\left(\theta^{2}\right),
\end{aligned}
$$

resulting in precisely the expression for the rotated vector. The full rotation is ${ }^{12}$

$$
\mathbf{U}=e^{-i \theta_{k} \boldsymbol{\sigma}_{k} / 2}=\cos \left(\frac{|\vec{\theta}|}{2}\right) \mathbf{1}-i \sin \left(\frac{|\vec{\theta}|}{2}\right) \frac{\theta_{k}}{|\vec{\theta}|} \boldsymbol{\sigma}_{k} .
$$

Thus, each rotation in $\mathrm{SO}(3)$ can be identified with a $2 \times 2$ unitary matrix with unit determinant, the group $\mathrm{SU}(2)$. However, both $\mathbf{U}$ and $\mathbf{-} \mathbf{U}$ generate the same rotation or, in other words, $\vec{\theta}$ and $-\vec{\theta}$ generate the same rotation, and thus, similar to the lift of $\mathbb{R} \mathbb{P}^{2}$ to $\mathbb{S}^{2}$, we have a sign ambiguity. Starting at $\vec{\theta}=0$, we can move along $\theta_{1}$, holding $\theta_{2}=$ $\theta_{3}=0$. In this case as $\theta_{1}$ goes from 0 to $2 \pi, \mathbf{U}(0,0,0)=$ $-\mathbf{U}(2 \pi, 0,0)$. We end up at the identical place in $\mathrm{SO}(3)$, but at a different place in $\mathrm{SU}(2)$. It follows that this loop is not contractible to a point and so the map from the measuring circuit to $\mathrm{SO}(3)$ which winds by $2 \pi$ is one of the nontrivial elements of $\pi_{1}[\mathrm{SO}(3)]$, traditionally denoted as $\mathbf{- 1}$.

Dividing out by $H_{b}$ yields additional defects akin to the stable nematic defects of charge $\frac{1}{2}$. In this case, however, there are $\pi$ rotations around each of $\hat{x}, \hat{y}$, and $\hat{z}$. Lifting those to $\mathrm{SU}(2)$, we find that they correspond to $\pm i \boldsymbol{\sigma}_{1}, \pm i \boldsymbol{\sigma}_{2}$, and $\pm i \sigma_{3}$, respectively, where the sign ambiguity corresponds to the same lifting ambiguity as above. These group elements are difficult to deal with; they do not commute, so a rotation

${ }^{12}$ To establish this exponential formula, consider the unitary matrix $\mathbf{S}$ which diagonalizes the Hermitian matrix $\mathbf{t}=\theta_{k} \boldsymbol{\sigma}_{k} / 2=$ $\mathbf{S} \boldsymbol{\Lambda} \mathbf{S}^{\dagger}$, where $\boldsymbol{\Lambda}$ is the diagonal matrix of eigenvalues. Then $\mathbf{T}=$ $\exp \{i \mathbf{t}\}=\exp \left\{i \mathbf{S} \boldsymbol{\Lambda} \mathbf{S}^{\dagger}\right\}=\mathbf{S} \exp \{i \boldsymbol{\Lambda}\} \mathbf{S}^{\dagger} . \quad$ Since $\operatorname{Tr} \boldsymbol{\Lambda}=\operatorname{Tr} \mathbf{t}=0$, $\boldsymbol{\Lambda}=\operatorname{diag}[\lambda,-\lambda]$, and it follows that

$$
\begin{aligned}
\mathbf{T} & =\mathbf{S}\left[\begin{array}{cc}
e^{i \lambda} & 0 \\
0 & e^{-i \lambda}
\end{array}\right] \mathbf{S}^{\dagger} \\
& =\cos (\lambda) \mathbf{1}+i \frac{\sin (\lambda)}{\lambda} \mathbf{S} \boldsymbol{\Lambda} \mathbf{S}^{\dagger}, \\
& =\cos (\lambda) \mathbf{1}+i \sin (\lambda) \frac{\mathbf{t}}{\lambda} .
\end{aligned}
$$

Since $-\lambda^{2}=\operatorname{det} \Lambda=\operatorname{det} \mathbf{t}=-|\vec{\theta}|^{2} / 4$, the identity follows. of $\pi$ around $\hat{x}$ followed by a rotation of $\pi$ around $\hat{y}$, followed by a rotation of $-\pi$ around $\hat{x}$ gives $\left[i \boldsymbol{\sigma}_{1}\right]^{-1}\left[i \boldsymbol{\sigma}_{2}\right]\left[i \boldsymbol{\sigma}_{1}\right]=$ $i \boldsymbol{\sigma}_{1} \boldsymbol{\sigma}_{2} \boldsymbol{\sigma}_{1}=-i \boldsymbol{\sigma}_{2}$. This means that the combination of paths on $\mathrm{SO}(3)$ that begin and end at one of the elements of $H_{b}$ do not commute and $\pi_{1}\left[\mathrm{SO}(3) / H_{b}\right]$ is non-Abelian.

What about point defects in a biaxial nematic? As it turns out, there are no point defects in the biaxial system, so $\pi_{2}\left[\mathrm{SO}(3) / H_{b}\right]=\{1\}$ is the trivial group of one element. To see this requires only a little more work and a theorem that we will not prove but is covered in standard texts (Hatcher, 2002), including the gold standard by Mermin (1979). First we ask what does SU(2) look like as a manifold? Fortuitously, we have the answer in the above algebra. Note that we can consider a general matrix depending on the four real parameters $\tilde{x}=\left(x_{0}, \vec{x}\right) \in \mathbb{R}^{4}$ :

$$
\mathbf{V}=x_{0} \mathbf{1}+i x_{k} \boldsymbol{\sigma}_{k}=\left[\begin{array}{cc}
x_{0}+i x_{3} & i x_{1}+x_{2} \\
i x_{1}-x_{2} & x_{0}-i x_{3}
\end{array}\right] \text {, }
$$

so $\operatorname{det} \mathbf{V}=x_{0}^{2}+|\vec{x}|^{2}$ is the squared length of the vector $\tilde{x}$ in four dimensions. Since all elements $\mathbf{U} \in \mathrm{SU}(2)$ are of this form and $\operatorname{det} \mathbf{U}=1$, we see that $\mathrm{SU}(2)$ can be identified with the three-dimensional sphere $\mathbb{S}^{3}$. So what do maps from $\mathbb{S}^{2}$ to $\mathbb{S}^{3}$ look like? They are all the same; just as $\pi_{1}\left(\mathbb{S}^{2}\right)$ vanishes because we can shrink every loop on the sphere to a point, so too can we shrink every two-sphere on a three-sphere to a point. Thus $\pi_{2}\left(\mathbb{S}^{3}\right)=\pi_{2}[\mathrm{SU}(2)]=\{1\}$. To calculate $\pi_{2}\left[\mathrm{SO}(3) / H_{b}\right]$, we need only use the theorem in which $\pi_{2}(G)=\pi_{2}(G / H)$ if $H$ is a discrete group. ${ }^{13}$ Note that we saw this with $\mathbb{S}^{2}$ and $\mathbb{R} \mathbb{P}^{2}$; they both shared the same $\pi_{2}$.

Precisely because the biaxial nematic does not support point defects, it serves to illuminate our previous discussion of disclination loops and their relation to hedgehogs. To see what we have gained it suffices to consider the simplest example: a disclination loop surrounding a single colloid in a Saturn ring configuration, illustrated in Fig. 10. As usual the meridional cycle of a torus sheathing the disclination records the nontrivial element of $\pi_{1}\left(\mathbb{R P}^{2}\right)$ and here, since the disclination is not linked with another, the longitudinal cycle records the trivial element. However, this cycle is still interesting. Note, in particular, that the director field on the inside of the torus (the part closest to the colloid) undergoes a $2 \pi$ rotation as we traverse this longitudinal cycle. Thus, if we were to decorate the uniaxial texture with a perpendicular short axis to produce a biaxial nematic then we would find that this cycle corresponded to a noncontractible loop in $\mathrm{SO}(3) / H_{b}$, recording the element $-\mathbf{1}$ of $\pi_{1}\left[\mathrm{SO}(3) / H_{b}\right]$. The Saturn ring was linked with another disclination after all: an all but invisible one only revealed by adding a small amount of biaxiality. This additional biaxial defect is not present for all defect loops: It is associated, as anticipated at the end of the last section, with a disclination loop that

\footnotetext{
${ }^{13}$ For the cognoscenti recall that there is a long exact sequence of homotopy groups$$
\cdots \rightarrow \pi_{2}(H) \rightarrow \pi_{2}(G) \rightarrow \pi_{2}(G / H) \rightarrow \pi_{1}(H) \rightarrow \cdots,
$$

and if $H$ is discrete then $\pi_{2}(H)=\pi_{1}(H)=\{1\}$, from which it follows that $\pi_{2}(G)=\pi_{2}(G / H)$.
} 


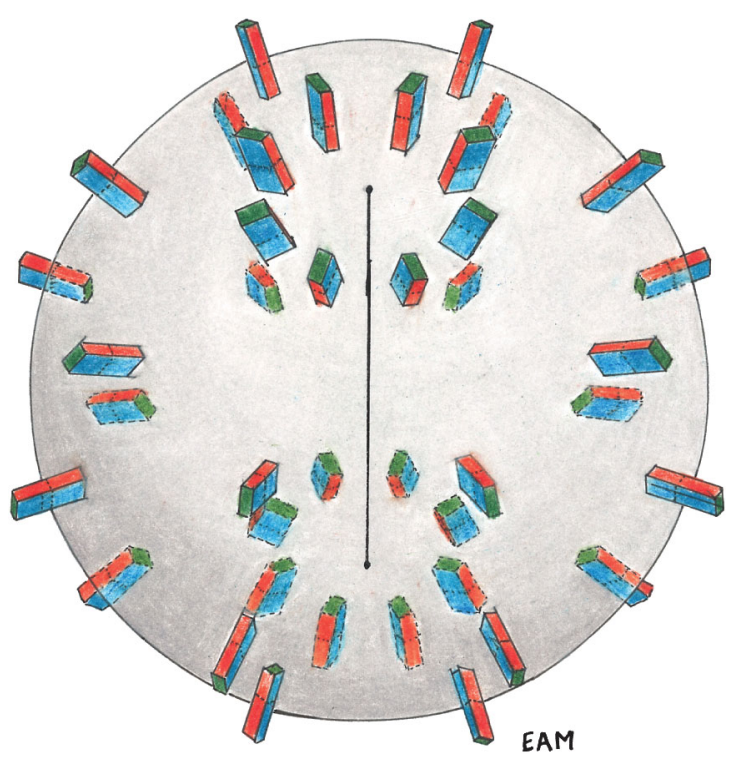

FIG. 10 (color). Schematic of biaxial order on a colloid with radial anchoring conditions for the long axis. The two shorter axes undergo a $2 \pi$ rotation at the North and South Poles and the particle is threaded by a $\mathbf{- 1}$ disclination denoted by a black line running between the two poles. It follows that the Saturn ring defect accompanying the colloid is linked with this biaxial disclination.

carries odd hedgehog charge, of which the Saturn ring is the simplest example.

It is useful to explore this connection a little further. Suppose we remove the colloid, leaving behind just the disclination loop and the texture on the torus surrounding it. Could we fill things back in differently, say without the hedgehog? Note that the configuration on the inside of the torus is just the same as on a cylindrical capillary with perpendicular anchoring. Thus to avoid a singularity the director will have to escape in the third dimension, just as it does in a capillary. However, if we have biaxial order then the singularity cannot be avoided; although the long axis is well defined along the capillary axis the two shorter axes are not and we have a $\mathbf{- 1}$ disclination. ${ }^{14}$ We assume that the texture is uniform at large distances so that this defect too closes up to form a loop. Technically, we are considering textures with defects on $\mathbb{S}^{3}$, the usual three-dimensional Euclidean space with a point at infinity added. The biaxial $\mathbf{- 1}$ disclination can also be surrounded by a torus, whose meridian records a $2 \pi$ winding and longitude records the $\pi$ winding of our original $\frac{1}{2}$ loop with which it is linked. Now by escaping in the third dimension the long axis can be made regular on any local section of the $\mathbf{- 1}$ defect, but this cannot be extended globally since the long axis is required to undergo a $\pi$ rotation along any path encircling the $\frac{1}{2}$ disclination. This $\pi$ rotation reverses the orientation of the director and converts an initial escape "up" into an escape "down" so that there must be a mismatch somewhere. Just as in the cylindrical capillary this mismatch marks the location of a point defect in the uniaxial nematic, the odd hedgehog.

\footnotetext{
${ }^{14}$ This transferral of winding from one axis to a perpendicular one is the Mermin-Ho relation (Mermin et al., 1976).
}

\section{CONCLUSION}

In the hope of avoiding to provide only a poor imitation of the many excellent reviews of the homotopy theory of defects already available (Mermin, 1979; Michel, 1980; Trebin, 1982; Kurik and Lavrentovich, 1988), we focused our discussion on a single physical system, nematic liquid crystal colloids, rather than provide a general survey and have eschewed, as far as possible, all of the formalities of algebraic topology.

A recurring issue is the necessity of a base point to induce the group structure on the set of homotopy classes. From a physical perspective the base point is really fiction, a convenience introduced for its useful computational attributes rather than because it conveys any deep significance. As mentioned in Sec. II the choice of base point for this purpose is entirely arbitrary so that any choice we make should yield the same results as any other. Fortunately, it is a simple exercise, proven in all of the standard reviews (Mermin, 1979), to show that the homotopy groups $\pi_{k}\left(\mathrm{GSM}, \Upsilon_{0}\right)$ and $\pi_{k}\left(\mathrm{GSM}, \mathrm{Y}_{0}^{\prime}\right)$, defined with base points $Y_{0}$ and $Y_{0}^{\prime}$, respectively, are isomorphic. The physical properties of defects should be independent of any choice of base point that we may make and the isomorphism between homotopy groups with different base points assures this. More importantly, however, a continuous deformation of a liquid crystal texture need not, in general, hold any point on any measuring circuit fixed while all others can freely vary. No physical distinction should be ascribed to defects that are nonhomotopic when a base point is used, but that become homotopic when all points are allowed to freely vary. This is an issue for hedgehogs where, as we saw in Sec. III, dragging a point defect around a disclination line required us to carefully keep track of the base point. Although at the end of the process the base point returned to its original value in $\mathbb{R} \mathbb{P}^{2}$, the journey was not uneventful and resulted in the reversal of the sign of the hedgehog charge. Thus while $d$ and $-d$ are distinct when a base point is held fixed, they may be freely converted into each other if all points on the measuring circuit are allowed to vary. Without the base point the group structure is lost and since, as we have argued, it is more natural to do without the base point, the combination of defects in condensed matter does not normally follow the laws of group composition ${ }^{15}$; cases such as the $X Y$ or Heisenberg models are exceptions to this norm. Of course the group composition can be regained, but only at the expense of keeping track of paths that the defects move along, the tethers of Sec. IV.

Throughout this Colloquium we avoided discussing any details of how the defects themselves appear and disappear. Indeed, when we have tried to observe liquid crystal textures under the microscope, we noted, upon cooling, the sudden appearance of many defects followed by a coarsening of defects too rapid for us to photograph. ${ }^{16}$ The coarsening is a situation in which the defects become dynamical objects. Not only do they move, they coalesce into defect and antidefect pairs and disappear. Although the addition rules

\footnotetext{
${ }^{15}$ This is even more vivid in systems with broken translational symmetry such as smectic liquid crystals (Chen, Alexander, and Kamien, 2009).

${ }^{16}$ This may reflect more the experimental abilities of the authors than the true nature of liquid crystals.
} 
afforded by the general homotopy group structure are preserved, the sample itself changes. Since defects are places in which the local order is ill defined, we can count the number of defects as well as measuring their charge. When defects annihilate the defect number changes. To account for this we have to perform "surgery" where we remove an arbitrarily small region of the sample along an incision and replace it with a new region that matches the texture smoothly along the cut. A proper formulation of this problem is beyond the scope of this review and will appear elsewhere (Chen et al.).

We intended to bring to light the subtleties of the theory of topological defects in experimentally realizable systems. With the advent of recent experimental work on colloidal inclusions in liquid crystals, these issues are no longer purely academic.

\section{ACKNOWLEDGMENTS}

We are grateful to Frederick Cohen, Simon Čopar, Tom Lubensky, Carl Modes, Miha Ravnik, and Jeffrey Teo for insightful discussions. We thank the School of Mathematics at the Institute for Advanced Study and the Aspen Center for Physics for their hospitality while this work was carried out. This work was supported by DMR05-47230.

\section{REFERENCES}

Abrikosov, A.A., 1957, "On the Magnetic Properties of Superconductors of the Second Group," Zh. Eksp. Teor. Fiz. 32, 1442 [JETP 5, 1174 (1957)].

Bechluft-Sachs, S., and M. Hien, 1999, "The Global Defect Index," Commun. Math. Phys. 202, 403.

Chaikin, P. M., and T. C. Lubensky, 1995, Principles of Condensed Matter Physics (Cambridge University Press, Cambridge, England).

Chen, B. G., G. P. Alexander, and R. D. Kamien, 2009, "Symmetry breaking in smectics and surface models of their singularities," Proc. Natl. Acad. Sci. U.S.A. 106, 15577.

Chen, B. G., G. P. Alexander, E. A. Matsumoto, and R. D. Kamien (unpublished).

Cladis, P. E., and M. Kléman, 1972, "Non-singular disclinations of strength $s=+1$ in nematics," J. Phys. (France) 33, 591.

Coleman, S., 1975, "Quantum sine-Gordon equation as the massive Thirring model," Phys. Rev. D 11, 2088.

Coleman, S., 1988, Aspects of Symmetry: Selected Erice Lectures (Cambridge University Press, Cambridge, England).

Čopar, S., and S. Žumer, 2011, "Nematic braids: topological invariants and rewiring of disclinations," Phys. Rev. Lett. 106, 177801.

Dupuis, A., D. Marenduzzo, and J. M. Yeomans, 2005, “Numerical calculations of the phase diagram of cubic blue phases in cholesteric liquid crystals," Phys. Rev. E 71, 011703.

Frank, F. C., 1958, "I. Liquid crystals. On the theory of liquid crystals," Faraday Discuss. 25, 19.

Grebel, H., R. M. Hornreich, and S. Shtrikman, 1983, "Landau theory of cholesteric blue phases," Phys. Rev. A 28, 1114.

Guzmán, O., E. B. Kim, S. Grollau, N. L. Abbott, and J. J. dePablo, 2003, "Defect Structure around Two Colloids in a Liquid Crystal," Phys. Rev. Lett. 91, 235507.

Harris, A. B., R. D. Kamien, and T. C. Lubensky, 1999, "Molecular chirality and chiral parameters," Rev. Mod. Phys. 71, 1745.
Hatcher, A., 2002, Algebraic Topology (Cambridge University Press, Cambridge, England).

Jänich, K., 1987, "Topological properties of ordinary nematics in 3-space," Acta Appl. Math. 8, 65.

Kamien, R. D., 2002, "The geometry of soft materials: A primer," Rev. Mod. Phys. 74, 953.

Kamien, R. D., 2011, “Knot your simple defect lines,” Science 333, 46.

Kitaev, A. Yu., 2003, "Fault-tolerant quantum computation by anyons," Ann. Phys. (N.Y.) 303, 2.

Kosterlitz, J. M., and D. J. Thouless, 1973, “'Ordering, metastability and phase transitions in two-dimensional systems," J. Phys. C, 6, 1181.

Kurik, M. V., and O.D. Lavrentovich, 1988, "Defects in liquid crystals: homotopy theory and experimental studies," Phys. Usp. 31, 196.

Lintuvuori, J.S., D. Marenduzzo, K. Stratford, and M.E. Cates, 2010, "Colloids in liquid crystals: a lattice Boltzmann study," J. Mater. Chem. 20, 10547.

Mermin, N. D., 1979, "The topological theory of defects in ordered media," Rev. Mod. Phys. 51, 591.

Mermin, N.D., and T.L. Ho, 1976, "Circulation and angular momentum in the $A$ phase of superfluid Helium-3," Phys. Rev. Lett. 36, 594; "Circulation and angular momentum in the $A$ phase of superfluid Helium-3," 36, 832(E) (1976).

Meyer, R. B., 1973, "On the existence of even indexed disclinations in nematic liquid crystals," Philos. Mag. 27, 405.

Michel, L., 1980, "Symmetry defects and broken symmetry. Configurations hidden symmetry," Rev. Mod. Phys. 52, 617.

Mühlbauer, S., B. Binz, F. Jonitez, C. Pfleiderer, A. Rosch, A. Neubauer, R. Georgii, and P. Böni, 2009, "Skyrmion Lattice in a Chiral Magnet," Science 323, 915.

Muševič, I., M. Škarabot, U. Tkalec, M. Ravnik, and S. Žumer, 2006, "Two-dimensional nematic colloidal crystals selfassembled by topological defects," Science 313, 954.

Nakanishi, H., K. Hayashi, and H. Mori, 1988, "Topological classification of unknotted ring defects," Commun. Math. Phys. 117, 203.

Nayak, C., S. H. Simon, A. Stern, M. Freedman, and S. Das Sarma, 2008, "Non-Abelian anyons and topological quantum computation," Rev. Mod. Phys. 80, 1083.

Nelson, D.R., 1988, “Vortex Entanglement in High- $T_{c}$ Superconductors," Phys. Rev. Lett. 60, 1973.

Poénaru, V., and G. Toulouse, 1977, "The crossing of defects in ordered media and the topology of 3-manifolds," J. Phys. (France) 38, 887.

Polyakov, A. M., 1974, "Particle spectrum in quantum field theory," ZhETF Pis. Red. 20, 430 [JETP 20, 194 (1974)] [http://www .jetpletters.ac.ru/ps/1789/article_27297.shtml].

Poulin, P., H. Stark, T. C. Lubensky, and D. A. Weitz, 1997, "Novel Colloidal Interactions in Anisotropic Fluids," Science 275, 1770.

Priest, R. G., and T. C. Lubensky, 1974, "Biaxial model of cholesteric liquid crystals," Phys. Rev. A 9, 893.

Ravnik, M., M. Škarabot, S. Žumer, U. Tkalec, I. Poberaj, D. Babič, N. Osterman, and I. Muševič, 2007, "Entangled Nematic Colloidal Dimers and Wires," Phys. Rev. Lett. 99, 247801.

Ravnik, M., and S. Žumer, 2009, "Nematic colloids entangled by topological defects," Soft Matter 5, 269.

Rößler, U.K., A.N. Bogdanov, and C. Pfleiderer, 2006, "Spontaneous skyrmion ground states in magnetic metals," Nature (London) 442, 797.

Škarabot, M., M. Ravnik, S. Žumer, U. Tkalec, I. Poberaj, D. Babič, N. Osterman, and I. Muševič, 2007, “Two-dimensional dipolar nematic colloidal crystals,” Phys. Rev. E 76, 051406. 
Škarabot, M., M. Ravnik, S. Žumer, U. Tkalec, I. Poberaj, D. Babič, N. Osterman, and I. Muševič, 2008, "Interactions of quadrupolar nematic colloids," Phys. Rev. E 77, 031705.

Smalyukh, I. I., Y. Lansac, N. A. Clark, and R.P. Trivedi, 2010, "Three-dimensional structure and multistable optical switching of triple-twisted particle-like excitations in anisotropic fluids," Nature Mater. 9, 139.

Terentjev, E. M., 1995, "Disclination loops, standing alone and around solid particles, in nematic liquid crystals," Phys. Rev. E 51, 1330.
Tkalec, U., M. Ravnik, S. Čopar, S. Žumer, and I. Muševič, 2011, "Reconfigurable knots and links in chiral nematic colloids," Science 333, 62.

Trebin, H.R., 1982, "The topology of non-uniform media in condensed matter physics," Adv. Phys. 31, 195.

Williams, C., and Y. Bouligand, 1974, "Fils et disinclinaisons dans un nématique en tube capillaire," J. Phys. (France) 35, 589.

Wright, D. C., and N.D. Mermin, 1989, "Crystalline liquids: the blue phases," Rev. Mod. Phys. 61, 385. 